\title{
Moving Frames and Singularities of Prolonged Group Actions
}

\author{
Peter J. Olver ${ }^{\dagger}$ \\ School of Mathematics \\ University of Minnesota \\ Minneapolis, MN 55455 \\ U.S.A. \\ olver@math . umn . edu \\ http: //www . math. umn. edu/ olver
}

\begin{abstract}
The prolongation of a transformation group to jet bundles forms the geometric foundation underlying Lie's theory of symmetry groups of differential equations, the theory of differential invariants, and the Cartan theory of moving frames. Recent developments in the moving frame theory have necessitated a detailed understanding of the geometry of prolonged transformation groups. This paper begins with a basic review of moving frames, and then focuses on the study of both regular and singular prolonged group orbits. Highlights include a corrected version of the basic stabilization theorem, a discussion of "totally singular points," and geometric and algebraic characterizations of totally singular submanifolds, which are those that admit no moving frame. In addition to applications to the method of moving frames, the paper includes a generalized Wronskian lemma for vector-valued functions, and methods for the solution to Lie determinant equations.
\end{abstract}

AMS subject Classification Numbers: 57SXX, 58A05, 58A20, 53A55.

Key words: moving frame, Lie group, jet, Lie matrix, homogeneous space

$\dagger$ Supported in part by NSF Grant DMS 98-03154.

May 24, 2010 


\section{Introduction.}

Given a finite-dimensional Lie transformation group acting on a manifold, there is an induced action on the associated jet bundles known as the prolonged group action. This action forms the foundation of the Lie theory of symmetry groups of differential equations $[\mathbf{3 0}, \mathbf{3 6}]$. Differential invariants appear as invariants of the prolonged group action $[\mathbf{2 5}, \mathbf{1 9}, \mathbf{3 1}]$. Furthermore, the range of applicability of the Cartan method of moving frames $[\mathbf{8}, \mathbf{9}, \mathbf{1 6}, \mathbf{2 0}]$, as reformulated and extended in $[\mathbf{1 2}, \mathbf{1 3}]$, is intimately tied to the geometry of the prolonged group action on jet spaces. A wide variety of additional applications, including symmetries of variational problems, equivariant bifurcation problems [15], conservation laws and invariant differential forms, group-invariant solutions, etc., all rely on this basic construction; see $[\mathbf{3 0}, \mathbf{3 1}]$ for details. The present paper is devoted to a detailed investigation into the geometry of the prolonged group action, with particular emphasis on the orbit structure, and how it relates to the group-theoretic geometry of submanifolds.

Since the dimensions of the prolonged group orbits increases with order, the maximal prolonged orbit dimension eventually stabilizes. The singular orbits are those of less than this maximal dimension. In the case of planar curves, the singular variety determined by all the singular orbits can be characterized by the vanishing of the classical Lie determinant $[\mathbf{2 6}, \mathbf{3 1}]$ and its generalizations. A point in the original manifold is called "totally singular" if every jet fiber sitting over it consists entirely of singular orbits. The existence of totally singular points has, apparently, not been noted in the literature before. In fact, we demonstrate that an analytic transformation group cannot have totally singular points, although there are elementary examples of smooth actions which allow such singularities. The basic stabilization theorem $[\mathbf{3 6}, \mathbf{3 1}]$ states that a group that acts "locally effectively on subsets," as defined below, will act locally freely on an open subset of jet spaces of sufficiently high order. Previous versions of the theorem omitted the phrase "on subsets," and hence do not apply at totally singular points. As an application, we establish a generalization of the classical Wronskian lemma that characterizes linearly dependent functions.

These results have immediate applications to Cartan's moving frame approach to the geometry, equivalence, and symmetry of submanifolds. A submanifold is called regular of order $n$ if its $n$-jet lies in the regular subset of jet space where the prolonged group orbits are of maximal dimension. The newly developed method of "moving coframes" $[\mathbf{1 2}, \mathbf{1 3}]$ shows that an $n^{\text {th }}$ order moving frame can be constructed if and only if the submanifold is regular at order $n$. Most submanifolds are regular at some sufficiently high order, and hence admit a moving frame. The exceptions are the "totally singular" submanifolds, whose $n$-jet (at a point) lies in the singular subset for all $n$. Totally singular submanifolds are the solutions to the classical Lie determinant equation, which is the simplest invariant differential equation associated with the given transformation group. A simple example is provided by the action of the special affine group that governs the equi-affine geometry of curves in the plane [19]. In this case, any straight line is a totally singular submanifold at all its points, while smooth curves which have infinite order contact with a straight line are totally singular at the point of contact. Any other curve will admit (at least locally) an equi-affine moving frame at some order $[\mathbf{1 3}]$, even though the classical equi-affine Frenet frame has order 3 and only applies to curves without inflection points $[\mathbf{1 9}, \mathbf{1 2}]$. 
The final part of the paper is devoted to the explicit characterization, both geometrical and algebraic, of totally singular submanifolds. In the analytic category, we prove that a submanifold is totally singular if and only if its isotropy subgroup does not act locally freely on it. A similar result holds for smooth submanifolds provided every point thereon is totally singular. Finally, we analyze the most important case, which is a transitive group action on a homogeneous space $M=G / H$. Here, the totally singular submanifolds are characterized algebraically, being related to the existence of "self $H$-normalizing subgroups." This leads to a direct algebraic construction of the solutions to the Lie determinant equation. Our results are all illustrated by a number of explicit examples of independent interest.

\section{Introduction to Moving Frames.}

Throughout this paper, $G$ will denote an $r$-dimensional Lie group ${ }^{\dagger}$ acting smoothly on an $m$-dimensional manifold $M$. In the classical Cartan theory, $G$ is a group of geometrical significance, e.g., the Euclidean group, equi-affine group, projective group, etc. However, the methods developed in [13] apply to completely general Lie group actions.

Definition 2.1. The isotropy subgroup of a subset $S \subset M$ is defined as

$$
G_{S}=\{g \in G \mid g \cdot S=S\} .
$$

The global isotropy subgroup of $S$ is

$$
G_{S}^{*}=\bigcap_{z \in S} G_{z}=\{g \in G \mid g \cdot s=s \text { for all } s \in S\},
$$

consisting of group elements that fix all points in $S$.

The basic definition of a moving frame is implicit in the works of Cartan [8] and first appears explicitly in Griffiths [16].

Definition 2.2. A moving frame is defined as a smooth $G$-equivariant map $\rho: M \rightarrow$ $G$.

In general, given actions of $G$ on $M$ and $N$, a map $\varphi: M \rightarrow N$ is called equivariant if $\varphi(g \cdot z)=g \cdot \varphi(z)$ for all $g \in G, z \in M$. In the case of a moving frame, there are two natural actions of $G$ on itself — by left multiplication $h \mapsto g \cdot h$ or by right multiplication $h \mapsto h \cdot g^{-1}$, leading to the concepts of left and right moving frames. All classical constructions lead to left moving frames, and so from now on we shall only deal with them. There are, however, occasions when a right moving frame is easier to compute. Fortunately, there is a simple connection between the two: if $\rho(z)$ is any left moving frame, then $\widetilde{\rho}(z)=\rho(z)^{-1}$ is a right moving frame, and conversely.

In practice, the construction of a globally-defined moving frame on all of $M$ is difficult, and one is content to determine a locally-defined version. The necessary and sufficient conditions for the local existence of a moving frame are readily established.

$\dagger$ The moving frame methods can be extended to infinite-dimensional pseudo-group actions [12] but for simplicity, we will only consider the finite-dimensional case here. 
Theorem 2.3. A moving frame exists in a neighborhood of a point $z \in M$ if and only if $G$ acts freely and regularly near $z$.

Recall that a group is said to act freely if the isotropy subgroup of any individual point $z_{0} \in M$ is trivial: $G_{z_{0}}=\{e\}$. The group acts regularly if all its orbits have the same dimension and, moreover, each point $z_{0}$ has a system of neighborhoods such that each orbit intersects in a pathwise connected subset, cf. [30]. If $G$ acts freely, then its orbits automatically all have the same dimension, namely the dimension $r$ of $G$ itself. The regularity condition is only to avoid such pathologies as the irrational flow on the torus, where the orbits return arbitrarily close to themselves. In general, the group orbits all have dimension equal to $r$ if and only if the action is locally free, which means that $G_{z_{0}}$ is a discrete subgroup of $G$ for each $z_{0} \in M$. Locally free group actions admit locally equivariant moving frames.

Of course, most interesting group actions, including all the geometrical examples mentioned above, are not (locally) free. For example, the Euclidean group E(2) does not act freely on $\mathbb{R}^{2}$ since any rotation fixes its center. The problem is, of course, that the space does not have a large enough dimension to admit $r$-dimensional orbits, which is required for freeness. Thus, a group can only act freely on a manifold whose dimension is at least that of $G$. Although such actions are not free, they are usually effective, which means that the only group element which fixes every point of $M$ is the identity, and so the global isotropy subgroup is trivial: $G_{M}^{*}=\{e\}$. Non-effective actions can always be made effective by the following simple device: The global isotropy subgroup $G_{M}^{*}$ forms a normal subgroup of $G$. Moreover, the quotient group $G / G_{M}^{*}$ acts effectively on $M$ in essentially the same manner as $G$ does, cf. [31]. Specifically, if $g \in G$ has image $\widetilde{g} \in G / G_{M}^{*}$ then one unambiguously defines $\widetilde{g} \cdot z=g \cdot z$ for $z \in M$. For example, the projective action $x \mapsto(\alpha x+\beta) /(\gamma x+\delta)$ of $\operatorname{GL}(2, \mathbb{R})$ on $\mathbb{R} \mathbb{P}^{1}$ is not effective since the multiples of the identity $\lambda \mathbb{1}$ act trivially. The effectively acting quotient group $\operatorname{PSL}(2, \mathbb{R})=\mathrm{GL}(2, \mathbb{R}) /\{\lambda \mathbb{1}\}$ is known as the projective linear group.

There are three important methods for converting a non-free, but effective action into a free action. The first is to consider the Cartesian product action of $G$ on several copies of the manifold, $M^{\times n}=M \times \cdots \times M$, whereby

$$
g \cdot\left(z_{1}, \ldots, z_{n}\right)=\left(g \cdot z_{1}, \ldots, g \cdot z_{n}\right) .
$$

For $n$ sufficiently large, the resulting action will typically become free on a suitable open subset of $M^{\times n}$. For example, the action of the planar Euclidean group $\operatorname{SE}(2)$ on $M=\mathbb{R}^{2}$ becomes free on the off-diagonal part $\left\{z_{1} \neq z_{2}\right\} \subset M \times M$ of the second Cartesian product, because the only planar Euclidean motion that fixes two distinct points is the identity. Invariants of such a Cartesian product action are known as joint invariants; $[\mathbf{1 2}, \mathbf{3 4}]$ discuss applications of moving frames to the classification of joint invariants, which has important consequences in computer vision [6].

A second method is to prolong the group action to the jet spaces $\mathrm{J}^{n}=\mathrm{J}^{n}(M, p)$ defined by $p$-dimensional submanifolds $N \subset M$ under the equivalence relation of $n^{\text {th }}$ order contact; see the following section and [31] for details. Jet space is the natural setting for Lie's theory of symmetry groups of differential equations, cf. $[\mathbf{3 0}, \mathbf{3 1}]$, and for Cartan's theory of moving frames in geometry $[\mathbf{8}, \mathbf{2 0}, \mathbf{1 3}]$. Since group transformations preserve contact, 
there is a naturally induced prolonged group action on $\mathrm{J}^{n}$. The basic stabilization theorem of Ovsiannikov states that, for $n$ sufficiently large, the prolonged action is locally free on an open subset of $\mathrm{J}^{n}$, and hence a locally equivariant moving frame can be constructed thereon. However, traditional statements of this result $[\mathbf{3 6}, \mathbf{3 1}]$ are not quite correct, and we shall return to this question in Section 6 .

Finally, a new, hybrid construction that combines the joint invariants and differential invariants in a common framework, called "multi-space," has been recently proposed [35]. This framework is based on the classical finite difference calculus from numerical analysis [27], and treats the jets of submanifolds as limits of discrete collections of points, thereby "blowing-up" the diagonal of the Cartesian product space to produce a jet space of the appropriate order. Indeed, the simplest example of the multi-space construction is the algebro-geometric blow-up, replacing a pair of coincident points by a tangent direction [17]. Application of the moving frame construction in multi-space produces invariant numerical approximations to differential invariants [6] of importance in the theory of geometric integration of differential equations $[\mathbf{5}, \mathbf{1 1}]$. Also the theory of joint differential invariants, also known as semi-differential invariants in computer vision [28], can be included in this general construction. The details are still under development.

In this paper, we shall only examine the second scenario, where the group acts on the jet bundle by prolongation. The construction of a moving frame relies on the fact that, for $n$ sufficiently large, the group acts freely and regularly on the jet space $\mathrm{J}^{n}$. In general this is not entirely correct, and so a detailed analysis of the geometry of the prolonged transformation group is required.

The explicit construction of moving frames relies on the method of "normalization," which lies at the heart of the Cartan approach to moving frames and equivalence problems $[\mathbf{8}, \mathbf{1 0}, \mathbf{1 9}, \mathbf{3 1}]$; an early version can be found in Killing $[\mathbf{2 2}]$. The key observation $[\mathbf{1 2}, \mathbf{1 3}]$ is that normalization amounts to the choice of a cross-section to the group orbits.

Definition 2.4. Let $G$ act regularly on the $m$-dimensional manifold $M$ with $s$ dimensional orbits. A (local) cross-section is an $(m-s)$-dimensional submanifold $K \subset M$ such that $K$ intersects each orbit transversally, at most once.

The most important are the coordinate cross-sections

$$
K=\left\{z_{1}=c_{1}, \ldots, z_{s}=c_{s}\right\}
$$

obtained by equating $s$ of the coordinates (which, by relabeling if necessary, can be taken to be the first $s$ coordinates) to constants. Of course, any cross-section can be (locally) converted into a coordinate cross-section by a suitable choice of local coordinates. The elements $k \in K$ of the cross-section can be viewed as "canonical forms" for general points $z \in M$, and their coordinates provide the invariant moduli for the group action. Since $K$ has dimension $m-s$, precisely $m-s$ of these invariant functions will be functionally independent and therefore provide us with a generating system of invariants. In the case of a coordinate cross-section $(2.2)$, the first $s$ coordinates of $k=\left(c_{1}, \ldots, c_{s}, z_{s+1}, \ldots, z_{m}\right)$ will be constant, and are hence trivial invariants, while the latter $m-s$ coordinates will provide the fundamental system of invariants, as we now describe. 


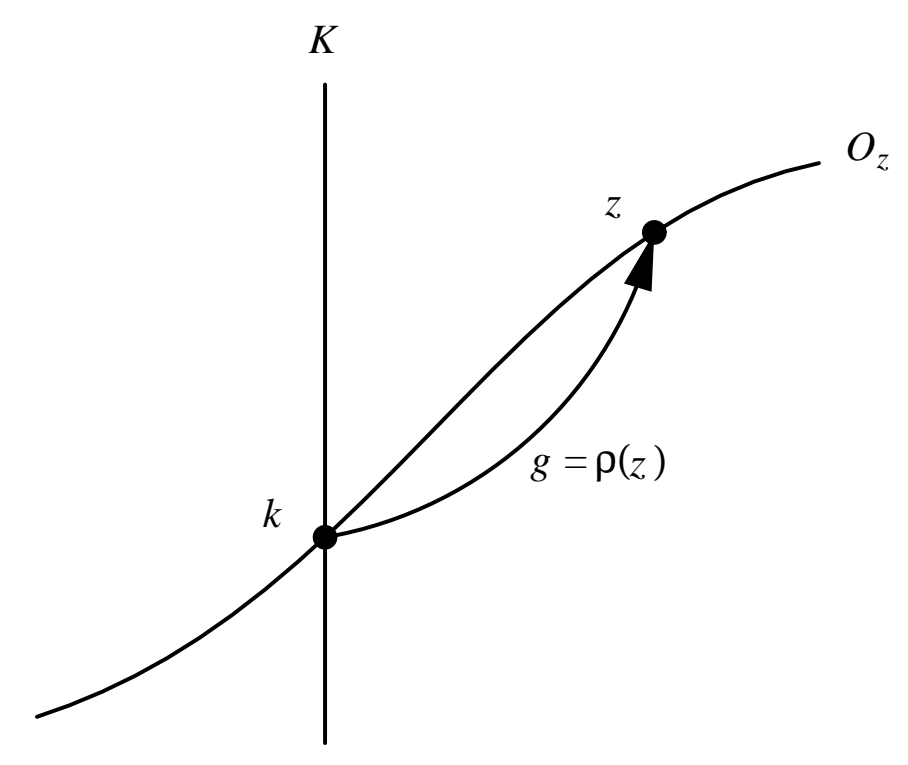

Figure 1. Moving Frame Associated with Cross-Section.

Theorem 2.5. Let $G$ act freely, regularly on $M$, and let $K$ be a cross-section to the group orbits. Given $z \in M$, let $k \in \mathcal{O}_{z} \cap K$ be the unique cross-section element lying in the orbit through $z$, and let $g=\rho(z)$ be the unique group element such that $g \cdot k=z$. Then $\rho: M \rightarrow G$ is a left moving frame for the group action.

Indeed, every moving frame has this form - the cross-section is just the inverse image of the identity: $K=\rho^{-1}\{e\}$. Therefore, to determine the moving frame associated with a chosen cross-section, one merely solves the normalization equations

$$
g^{-1} \cdot z=k \in K \quad \text { for } \quad g=\rho(z) .
$$

For example, if we choose the coordinate cross-section (2.2), and write out the group transformations in coordinates as $w(g, z)=g^{-1} \cdot z$, then the moving frame is obtained by solving the implicit equations

$$
w_{1}(g, x)=c_{1}, \quad \cdots \quad w_{r}(g, x)=c_{r},
$$

for the group parameters $g$ in terms of the coordinates $x$. Moreover, the fundamental invariants are found by substituting the resulting moving frame formula into the remaining, unnormalized components of $w(g, z)$.

Theorem 2.6. Given a free, regular Lie group action and coordinate cross-section as described above, let $g=\rho(x)$ denote the solution to the normalization equations (2.4). Then the functions

$$
I_{1}(x)=w_{r+1}(\rho(x), x), \quad \ldots \quad I_{m-r}(x)=w_{m}(\rho(x), x),
$$


obtained by substituting the moving frame formulae into the remaining $m-r$ components of $w$ form a complete system of functionally independent invariants for the action of $G$.

\section{Jet Bundles and Prolongation.}

Before presenting examples of moving frames, we need to review the basics of jet bundles and prolonged group actions. This will help prepare us for the more detailed investigations into their geometry and singularities. Given a manifold $M$, we let $\mathrm{J}^{n}=$ $\mathrm{J}^{n}(M, p)$ denote the $n^{\text {th }}$ order (extended) jet bundle consisting of equivalence classes of regular (embedded) $p$-dimensional submanifolds $S \subset M$ under the equivalence relation of $n^{\text {th }}$ order contact, cf. [30; Chapter 3$]$. In particular, $\mathrm{J}^{0}=M$. The $n^{\text {th }}$ jet space $\mathrm{J}^{n}$ is a fiber bundle $\pi_{0}^{n}: \mathrm{J}^{n} \rightarrow M$ of total dimension

$$
q^{(n)}=p+q\left(\begin{array}{c}
p+n \\
n
\end{array}\right)=\operatorname{dim} \mathrm{J}^{n}(M, p),
$$

whose fibers are generalized Grassmann manifolds, cf. [29]. We let $\mathrm{j}_{n} S \subset \mathrm{J}^{n}$ denote the $n$-jet of the submanifold $S$, which forms a $p$-dimensional submanifold of $\mathrm{J}^{n}$. Sometimes, it is convenient to work with the infinite jet bundle $\mathrm{J}^{\infty}=\mathrm{J}^{\infty}(M, p)$, which is defined as the inverse limit of the finite order jet bundles under the standard projections $\pi_{n}^{k}: \mathrm{J}^{k} \rightarrow \mathrm{J}^{n}$, $k>n$, with $\mathrm{j}_{\infty} S$ denoting the corresponding infinite jet of $S$. An analytic submanifold is uniquely determined by its infinite jet at a point in the same manner that an analytic function is uniquely determined by its Taylor series at a point.

We introduce local coordinates $z=(x, u)$ on $M$, considering the first $p$ components $x=$ $\left(x^{1}, \ldots, x^{p}\right)$ as independent variables, and the latter $q=m-p$ components $u=\left(u^{1}, \ldots, u^{q}\right)$ as dependent variables. Splitting the coordinates into independent and dependent variables has the effect of locally identifying $M$ with an open subset of a bundle $E=X \times U \simeq \mathbb{R}^{p} \times \mathbb{R}^{q}$. Sections $u=f(x)$ of $E$ correspond to $p$-dimensional submanifolds $S$ that are transverse to the vertical fibers $U_{c}=\{c\} \times U \subset E$. The induced local coordinates on the jet bundle $\mathrm{J}^{n}$ are denoted by $z^{(n)}=\left(x, u^{(n)}\right)$, with components $u_{J}^{\alpha}$ representing the partial derivatives of the dependent variables with respect to the independent variables up to order $n$. Here $J=$ $\left(j_{1}, \ldots, j_{k}\right)$, where $1 \leq j_{\nu} \leq p$, will denote a symmetric multi-index of order $k=\# J$. The $\left(x, u^{(n)}\right)$ define local coordinates on the open, dense subbundle $\mathrm{J}^{n} E \subset \mathrm{J}^{n}(M, p)$ determined by the jets of transverse submanifolds, or, equivalently, local sections $S=\{u=f(x)\}$ of $E$. Let $\mathrm{j}_{n} f(x)$ denote the corresponding $n$-jet of $f$ at $x$, which can be identified with the $n^{\text {th }}$ order Taylor polynomial of $f$ at $x$. In the limit, we let $z^{(\infty)}=\left(x, u^{(\infty)}\right)$ denote the corresponding coordinates on $\mathrm{J}^{\infty} E \subset \mathrm{J}^{\infty}(M, p)$, consisting of independent variables $x^{i}$, dependent variables $u^{\alpha}$, and their derivatives $u_{J}^{\alpha}, \alpha=1, \ldots, q$, of arbitrary order $\# J \geq 0$.

Any transformation group $G$ acting on $M$ preserves the order of contact between submanifolds. Therefore, there is an induced action of $G$ on the $n^{\text {th }}$ order jet bundle $\mathrm{J}^{n}=\mathrm{J}^{n}(M, p)$ known as the $n^{\text {th }}$ prolongation of $G$, and denoted by $G^{(n)}$. Note that if $G$ acts globally on $M$, then its prolonged action $G^{(n)}$ is also a global transformation group on $\mathrm{J}^{n}$, but, generally only a local transformation group on the coordinate subbundles $\mathrm{J}^{n} E$ since $G$ may not preserve transversality.

Remark: We shall exclusively deal with point transformation groups, although all our results and methods naturally extend to contact transformation groups, cf. [31]. 
The explicit local coordinate formulae for the prolonged group actions are found by implicit differentiation. Let us see how this works in a simple example.

Example 3.1. The planar Euclidean group $\mathrm{SE}(2)=\mathrm{SO}(2) \ltimes \mathbb{R}^{2}$ acts transitively on $M=\mathbb{R}^{2}$, mapping a point $(x, u)$ to

$$
y=x \cos \theta-u \sin \theta+a, \quad v=x \sin \theta+u \cos \theta+b,
$$

where $\theta, a, b$ serve to parametrize the group. The second order prolongation maps a point $\left(x, u, u_{x}, u_{x x}\right) \mapsto\left(y, v, v_{y}, v_{y y}\right)$, where $y, v$ are given by (3.2) and

$$
v_{y}=\frac{d v}{d y}=\frac{\sin \theta+u_{x} \cos \theta}{\cos \theta-u_{x} \sin \theta}, \quad v_{y y}=\frac{d^{2} v}{d y^{2}}=\frac{u_{x x}}{\left(\cos \theta-u_{x} \sin \theta\right)^{3}} .
$$

The basic moving frame construction for a free prolonged group action proceeds as in Theorem 2.6. Solving the normalization equations for $r=\operatorname{dim} G$ components of the prolonged transformation formulae

$$
w^{(n)}\left(g^{(n)}, z^{(n)}\right)=\left(g^{(n)}\right)^{-1} \cdot z^{(n)}
$$

results in a moving frame on the $n^{\text {th }}$ order jet space. In classical situations, the resulting equivariant map $\rho: \mathrm{J}^{n} \rightarrow G$ can be identified with the standard geometric moving frame. We will illustrate the basic procedure with two well-studied examples.

Example 3.2. The prolonged planar Euclidean group $\mathrm{SE}(2)$ action is (globally) free on $\mathrm{J}^{1}$, and hence also on any $\mathrm{J}^{n}$ for $n \geq 1$. According to Theorem 2.6, we can construct a left moving frame by normalizing the inverse group transformations ${ }^{\dagger}$

$$
\begin{aligned}
y & =\cos \theta(x-a)+\sin \theta(u-b), & v & =-\sin \theta(x-a)+\cos \theta(u-b), \\
v_{y} & =\frac{u_{x} \cos \theta-\sin \theta}{\cos \theta+u_{x} \sin \theta}, & v_{y y} & =\frac{u_{x x}}{\left(\cos \theta+u_{x} \sin \theta\right)^{3}} .
\end{aligned}
$$

Consider the simple coordinate cross-section $K=\left\{x=u=u_{x}=0\right\}$. The solution to the associated normalization equations

$$
y=0, \quad v=0, \quad v_{y}=0,
$$

produces the classical moving frame $\rho: \mathrm{J}^{1} \rightarrow \mathrm{SE}(2)$, with

$$
\theta=\tan ^{-1} u_{x}, \quad a=x, \quad b=u .
$$

Indeed, in its standard matrix form

$$
\left(\begin{array}{cc}
\cos \theta & -\sin \theta \\
\sin \theta & \cos \theta
\end{array}\right)=\frac{1}{\sqrt{1+u_{x}^{2}}}\left(\begin{array}{cc}
1 & -u_{x} \\
u_{x} & 1
\end{array}\right), \quad\left(\begin{array}{l}
a \\
b
\end{array}\right)=\left(\begin{array}{l}
x \\
u
\end{array}\right)
$$

$\dagger$ Normalization of the group transformations (3.2), (3.3) leads to a right moving frame. The invariants are constructed as in the left version. 
the columns of the rotation component represent the unit tangent and unit normal to the curve, while the translation component provides the point on the curve. Substituting the normalizations (3.5) into the final formula in (3.4) for the second order derivative $v_{y y}$, we recover the fundamental Euclidean curvature invariant

$$
\kappa=\frac{u_{x x}}{\left(1+u_{x}^{2}\right)^{3 / 2}} .
$$

If we prolong further, say to $\mathrm{J}^{4}$, and substitute the moving frame formulae (3.5) into the corresponding formulae for the third and fourth derivatives, we obtain the higher order differential invariants

$$
v_{y y y} \longmapsto \frac{d \kappa}{d s}, \quad v_{y y y y} \longmapsto \frac{d^{2} \kappa}{d s^{2}}-3 \kappa^{3},
$$

where $d / d s$ denotes differentiation with respect to the Euclidean arc length element

$$
d s=\sqrt{1+u_{x}^{2}} d x
$$

The Euclidean arc length (3.8) is characterized as the unique, up to constant multiple, Euclidean-invariant one-form of lowest order. More correctly, it is a "contact-invariant" horizontal one-form on the jet bundle $\mathrm{J}^{1}$, cf. [13]. It is obtained by the general procedure of substituting the moving frame normalizations (3.5) into the horizontal (or total) differential $d y=\left(\cos \theta+u_{x} \sin \theta\right) d x$ of the chosen independent variable.

Remark: A general result [13] states that all the higher order differential invariants are obtained by differentiating the fundamental differential invariants — in this case the curvature $\kappa$ - with respect to to the invariant one-forms - in this case arc length $d s$. The fact that the normalized higher order invariants (3.7) do not in general agree with the differentiated invariants is of critical important in understanding the syzygies (functional relationships) among the differential invariants. A general algorithm for determining the "correction terms" — for example, the $-3 \kappa^{3}$ in the second formula in (3.7) — was found in $[\mathbf{1 3}, \mathbf{1 4}]$, and applied to provide a complete solution to the syzygy classification problem for differential invariants.

The Euclidean group is atypical in that its first prolongation acts freely everywhere on the jet spaces $\mathrm{J}^{n}, n \geq 1$. More typically, the prolonged actions are free (if at all) only on a dense open subset of the jet space. A more representative example follows.

Example 3.3. The equi-affine geometry of curves in the plane is governed by the standard action

$$
(x, u) \longmapsto(\alpha x+\beta u+a, \gamma x+\delta u+b), \quad \alpha \delta-\beta \gamma=1,
$$

of the special affine group $\mathrm{SA}(2)=\mathrm{SL}(2) \ltimes \mathbb{R}^{2}$ acting on $M=\mathbb{R}^{2}$, cf. [7, 19]. The components of $w=g^{-1} \cdot(x, u)$ are

$$
y=\delta(x-a)-\beta(u-b), \quad v=-\gamma(x-a)+\alpha(u-b) .
$$


Since the group has dimension 5 , we need to prolong to $\mathrm{J}^{3}$ at least to have any chance of the prolonged action being free, and the first differential invariant should appear on $\mathrm{J}^{4}$. The explicit formulae for the fourth prolongation are

$$
\begin{gathered}
v_{y}=-\frac{\gamma-\alpha u_{x}}{\delta-\beta u_{x}}, \quad v_{y y}=-\frac{u_{x x}}{\left(\delta-\beta u_{x}\right)^{3}}, \quad v_{y y y}=-\frac{\left(\delta-\beta u_{x}\right) u_{x x x}+3 \beta u_{x x}^{2}}{\left(\delta-\beta u_{x}\right)^{5}}, \\
v_{y y y y}=-\frac{\left(\delta-\beta u_{x}\right)^{2} u_{x x x x}+10 \beta\left(\delta-\beta u_{x}\right) u_{x x} u_{x x x}+15 \beta^{2} u_{x x}^{3}}{\left(\alpha+\beta u_{x}\right)^{7}} .
\end{gathered}
$$

The action is free on the open subset $\mathcal{V}^{4}=\left\{u_{x x} \neq 0\right\} \subset \mathrm{J}^{4}$, and hence a local moving frame can be constructed. Thus we recover the classical result, that a curve $u=f(x)$ admits an equi-affine moving frame of order 3 if and only if it does not have an inflection point: $f^{\prime \prime}(x) \neq 0$. Indeed, choosing the normalizations

$$
y=0, \quad v=0, \quad v_{y}=0, \quad v_{y y}=1, \quad v_{y y y}=0,
$$

we obtain the classical third order equi-affine moving frame $\rho: \mathrm{J}^{3} \rightarrow \mathrm{SA}(2)$, given by

$$
\left(\begin{array}{cc}
\alpha & \beta \\
\gamma & \delta
\end{array}\right)=\left(\begin{array}{cc}
\sqrt[3]{u_{x x}} & -\frac{1}{3} u_{x x}^{-5 / 3} u_{x x x} \\
u_{x} \sqrt[3]{u_{x x}} & u_{x x}^{-1 / 3}-\frac{1}{3} u_{x x}^{-5 / 3} u_{x x x}
\end{array}\right), \quad\left(\begin{array}{l}
a \\
b
\end{array}\right)=\left(\begin{array}{l}
x \\
u
\end{array}\right) .
$$

The columns of the unimodular matrix can be identified as the equi-affine tangent and normal to the curve [7], while the translational component is the point on the curve. The first differential invariant is found by inserting the moving frame normalizations into the next component $v_{y y y y}$, leading to the equi-affine curvature

$$
\kappa=\frac{3 u_{x x} u_{x x x x}-5 u_{x x x}^{2}}{3 u_{x x x}^{8 / 3}} .
$$

The equi-affine arc length

$$
d s=\sqrt[3]{u_{x x}} d x
$$

is obtained, as above, by normalizing $d y=\left(\alpha+\beta u_{x}\right) d x$. As in the Euclidean case, all higher order differential invariants are obtained by differentiation of the curvature with respect to the arc length.

The classical restriction of the moving frame method to equi-affine curves without inflection points can, in fact, be circumvented by using to a higher order prolongation. Indeed, the fifth order prolongation of (3.9) acts freely on $\mathcal{V}^{5}=\left\{u_{x x} u_{x x x} \neq 0\right\} \subset J^{5}$, and hence a fifth order equi-affine moving frame can, in principle, be constructed for any curve $u=f(x)$ without a second order inflection point, i.e., provided either $f^{\prime \prime}(x)$ or $f^{\prime \prime \prime}(x) \neq 0$. (Unfortunately, the actual normalizations require the solution of a fifth degree polynomial equation, and hence the explicit formulae for a fifth order moving frame are not available.) More generally, $\mathrm{SA}(2)$ acts freely on the regular subset $\mathcal{V}^{n}=\mathrm{J}^{n} \backslash \mathcal{S}^{n}, n \geq 3$, where

$$
\mathcal{S}^{n}=\left\{u_{x x}=u_{x x x}=\cdots=u_{k}=0\right\}, \quad k=1+\left[\frac{n}{2}\right],
$$

is the singular subset, and we abbreviate the $k^{\text {th }}$ order derivative of $u$ with respect to $x$ by $u_{k}=\left(D_{x}\right)^{k} u$. Therefore, every curve admits a (local) equi-affine moving frame of 
some sufficiently high order unless it has an inflection point of infinite order: $f^{(n)}(x)=0$, $n=2,3,4, \ldots$. Curves that do not admit moving frames will be called totally singular, and their geometric characterization forms the focus of the final part of this paper. In particular, the only analytic totally singular curves under the equi-affine group are the straight lines $u=m x+l$.

Once the moving frame has been constructed and the corresponding differential invariants classified, a complete solution to the equivalence problem - when can two submanifolds be mapped to each other by a group transformation - is effected by considering the signature manifold which is parametrized by the fundamental differential invariants. In the case of Euclidean or equi-affine curves, the signature curve is parametrized by the first two differential invariants $\left(\kappa, \kappa_{s}\right)$. Two curves are equivalent if and only if their signature curves are identical. We refer the reader to [13] for details, including a variety of higher dimensional cases. Applications to classical invariant theory and the equivalence and symmetry properties of binary forms are discussed in $[33,4]$. Applications to computer vision and the recognition of objects in images can be found in [6]. Applications to the design of invariant numerical algorithms can be found in [35].

\section{Stabilization and Orbit Dimensions.}

The theoretical foundations of the moving frame method outlined in the preceding section motivate a more detailed discussion of the geometry of the higher order prolongations of a transformation group. The key questions that need to be addressed are:

( $i$ ) Does the prolonged group action become free and regular, at least on an open subset of $\mathrm{J}^{n}$ at sufficiently high order $n \gg 0$ ? As the equi-affine example makes clear, one cannot expect the action to ever become free everywhere on $\mathrm{J}^{n}$.

(ii) How does one characterize the singular subset $\mathcal{S}^{n} \subset \mathrm{J}^{n}$ where the action is not free?

(iii) How does one characterize the totally singular submanifolds, meaning those whose $n$-jets have nonempty intersection with the singular subset $\mathcal{S}^{n}$ for all $n$ ?

Since these questions rely on the structure of the prolonged group orbits, the first order of business is to understand their dimensions. In particular, the "generic" orbits those of maximal dimension — have a distinguished status.

Definition 4.1. Given $G$ acting on $M$, let $s_{n}$ denote the maximal orbit dimension of the prolonged action $G^{(n)}$ on $\mathrm{J}^{n}$. The stable orbit dimension of $G$ is defined to be $s=\max s_{n}$. The stabilization order of $G$ is the minimal $n$ such that $s_{n}=s$.

Remark: Since the prolonged orbits map onto each other by the standard jet projections $\pi_{k}^{n}: \mathrm{J}^{n} \rightarrow \mathrm{J}^{k}, k<n$, the orbit dimensions are clearly nondecreasing:

$$
s_{0} \leq s_{1} \leq \ldots \leq s_{n-1}<s_{n}=s_{n+1}=\cdots=s,
$$

where $n$ is the stabilization order. The group is said to pseudostabilize if $s_{k}=s_{k+1}<s$ for some $k<n$. In [31; Theorem 5.37], it was proved that a group can admit at most one pseudostabilization; in other words, if $s_{k}=s_{k+1}$ and $s_{n}=s_{n+1}$ for some $k<n$, then $s_{n}=s$, and hence the group stabilizes at order $n$ (or less). As a consequence, we immediately deduce a bound on the stabilization order. 
Theorem 4.2. The stabilization order $n$ of an $r$-dimensional transformation group is bounded by $n \leq r$. If the group does not pseudostabilize, this bound can be reduced to $n \leq r-1$.

Remark: There are very few examples of groups that pseudostabilize. In [32], I proved that every Lie group that pseudostabilizes at order zero is locally equivalent to the elementary $(m+1)$-dimensional similarity group $z \mapsto \lambda z+a$ parametrized by $\lambda \in \mathbb{R}$, $a \in \mathbb{R}^{m}$. Lie's classification of all nonsingular transformation groups in the plane $[\mathbf{2 4}, \mathbf{3 1}]$ provides examples of groups that pseudostabilize at higher order, and lead one to conjecture on the general form of such groups in higher dimensions. However, a general classification of pseudostabilizing transformation groups remains open. The short list of examples of pseudostabilizing group actions leads one to conjecture that the stabilization order is, in fact, always bounded by $r-1$, which is optimal, as demonstrated by the following example.

Example 4.3. Let

$$
h_{\kappa}(t)=\frac{d^{\kappa-1} a}{d t^{\kappa-1}} \varphi(t), \quad \kappa=1, \ldots, r,
$$

where $\varphi: \mathbb{R} \rightarrow \mathbb{R}^{q}$ is a fixed nonzero vector function, and $a(t)$ is any scalar function such that it and its first $r-1$ derivatives are linearly independent, i.e., $a$ is not the solution to a linear, constant coefficient ordinary differential equation of order $r-1$. Consider the $r$-parameter abelian group

$$
(x, u) \longmapsto\left(x, u+\sum_{\kappa=1}^{r} t_{\kappa} h_{\kappa}\left(x^{1}\right)\right), \quad \begin{aligned}
x & =\left(x^{1}, \ldots, x^{p}\right) \in \mathbb{R}^{p}, \\
u & =\left(u^{1}, \ldots, u^{q}\right) \in \mathbb{R}^{q}, \\
t & =\left(t_{1}, \ldots, t_{r}\right) \in G=\mathbb{R}^{r} .
\end{aligned}
$$

The orbits of $G$ are the vertical lines $\{(x, u+s \varphi(x)) \mid s \in \mathbb{R}\}$. Moreover, the prolonged orbit dimensions of the group (4.2) only increase by one at each order: $s_{k}=k+1$ for $k=0, \ldots, r-1$. Consequently, the stabilization order for (4.2) is $n=r-1$.

Definition 4.4. The regular subset $\mathcal{V}^{n} \subset \mathrm{J}^{n}$ is the open subset consisting of all prolonged group orbits of dimension equal to the stable orbit dimension. A point $z^{(n)} \in \mathrm{J}^{n}$ will be called a regular jet provided $z^{(n)} \in \mathcal{V}^{n}$. The remainder constitutes the singular subset $\mathcal{S}^{n}=\mathrm{J}^{n} \backslash \mathcal{V}^{n}$, which is the union of orbits of less than maximal dimension.

Note that, by this definition, $\mathcal{V}^{n}=\varnothing$ and $\mathcal{S}^{n}=\mathrm{J}^{n}$ if $n$ is less than the stabilization order of $G$, so that only jets of sufficiently high order can be regular. If $G$ acts analytically, then $\mathcal{V}^{n}$ is a dense open subset of $\mathrm{J}^{n}$ for $n$ greater than or equal to the stabilization order.

Remark: Suppose $W \subset M$ is an open submanifold. For smooth actions, it may well happen that $s(W)<s(M)$, where $s(W)$ denotes the stable orbit dimension for the restricted action of $G$ on $W$. For example, let

$$
\mathbf{h}_{0}(x)= \begin{cases}e^{-1 / x}, & x>0 \\ 0, & x \leq 0 .\end{cases}
$$


The one-parameter group

$$
(x, u) \mapsto\left(x, u+t \mathbf{h}_{0}(x)\right), \quad t \in G=\mathbb{R},
$$

acting on $M=\mathbb{R}^{2}$ has $s(M)=1$, while $s(W)=0$ when $W \subset\{x<0\}$ because $G$ acts completely trivially on the left half plane. As we shall see, such pathology cannot appear in the analytic category, where $s(W)=s(M)$ for all open $W \subset M$.

\section{Infinitesimal Generators.}

Lie's great discovery was that local geometry of a continuous, connected transformation group is entirely determined by its infinitesimal action. The space of infinitesimal generators of a transformation group $G$ forms a Lie algebra of vector fields on the manifold $M$, which, assuming $G$ acts locally effectively, can be identified with its Lie algebra $\mathfrak{g}$. In terms of local coordinates $(x, u)$ on $M$, the infinitesimal generators have the form

$$
\mathbf{v}=\sum_{i=1}^{p} \xi^{i}(x, u) \frac{\partial}{\partial x^{i}}+\sum_{\alpha=1}^{q} \varphi^{\alpha}(x, u) \frac{\partial}{\partial u^{\alpha}} .
$$

Let $\mathfrak{g}^{(n)}$ denote the corresponding Lie algebra of infinitesimal generators of the $n^{\text {th }}$ prolonged action $G^{(n)}$ on $\mathrm{J}^{n}$. Explicit local coordinate formulae for the prolonged infinitesimal generators are given by the well-known prolongation formula $[\mathbf{3 0 , 3 1}]$.

Definition 5.1. The characteristic of the vector field $\mathbf{v}$ given in (5.1) is the $q$-tuple $Q=\left(Q^{1}, \ldots, Q^{q}\right)$ of functions

$$
Q^{\alpha}\left(x, u^{(1)}\right)=\varphi^{\alpha}(x, u)-\sum_{i=1}^{p} \xi^{i}(x, u) u_{i}^{\alpha}, \quad \alpha=1, \ldots, q .
$$

A vector field $\mathbf{v}$ is tangent to a $p$-dimensional submanifold $S$ if and only if its characteristic vanishes on $S$. Therefore, the characteristic uniquely determines the submanifolds that are invariant under the induced one-parameter group, cf. [31; Theorem 4.9].

Proposition 5.2. The Lie algebra $\mathfrak{g}_{S}$ of the isotropy subgroup $G_{S}$ of a closed $p$ dimensional submanifold $S \subset M$ consists of all infinitesimal generators $\mathbf{v} \in \mathfrak{g}$ whose characteristics vanish identically on $S$.

Let $\mathbf{v} \in \mathfrak{g}$ generate the one-parameter subgroup $\exp (t \mathbf{v})$. The $n^{\text {th }}$ prolongation $\mathrm{pr}^{(n)} \mathbf{v}$ is the infinitesimal generator of the prolonged one-parameter group $\exp (t \mathbf{v})^{(n)}$ on $\mathrm{J}^{n}$. In terms of local coordinates $\left(x, u^{(\infty)}\right)$ on $\mathrm{J}^{\infty}$, we obtain $\mathrm{pr}^{(n)} \mathbf{v}$ by truncating the infinitely prolonged vector field

$$
\operatorname{pr} \mathbf{v}=\sum_{i=1}^{p} \xi^{i}(x, u) \frac{\partial}{\partial x^{i}}+\sum_{\alpha=1}^{q} \sum_{k=\# J \geq 0} \varphi_{J}^{\alpha}\left(x, u^{(k)}\right) \frac{\partial}{\partial u_{J}^{\alpha}}
$$

at order $n$. The coefficient functions $\varphi_{J}^{\alpha}$ of $\operatorname{pr} \mathbf{v}$ are explicitly constructed as follows. Let

$$
D_{i}=\frac{\partial}{\partial x^{i}}+\sum_{\alpha=1}^{q} \sum_{\# J \geq 0} u_{J, i}^{\alpha} \frac{\partial}{\partial u_{J}^{\alpha}}
$$


denote the usual total derivative with respect to $x^{i}$. Note that we can regard $D_{i}$ as a vector field on $\mathrm{J}^{\infty} E$, but not on any finite order jet bundle.

Definition 5.3. A total vector field on $\mathrm{J}^{\infty}$ is a linear combination $\sum_{i} P_{i}\left(x, u^{(n)}\right) D_{i}$ of total derivatives.

Remark: In fact, the characterization of total vector fields does not depend on the choice of independent and dependent variables. Indeed, as discussed in [31], they are the sections of the annihilator of the ideal generated by the contact forms.

Theorem 5.4. The prolonged vector field (5.3) can be uniquely written as a sum

$$
\operatorname{pr} \mathbf{v}=\mathbf{v}_{t o t}+\mathbf{v}_{e v},
$$

where

$$
\mathbf{v}_{t o t}=\pi_{t o t}(\operatorname{pr} \mathbf{v})=\sum_{i=1}^{p} \xi^{i}(x, u) D_{i}
$$

is the total vector field induced by the horizontal component of $\mathbf{v}$, and

$$
\mathbf{v}_{e v}=\pi_{e v}(\operatorname{pr} \mathbf{v})=\sum_{\alpha=1}^{q} \sum_{\# J \geq 0} D_{J} Q^{\alpha} \frac{\partial}{\partial u_{J}^{\alpha}}
$$

denotes the evolutionary vector field induced $\mathbf{v}$. Here $D_{J}=D_{j_{1}} \cdots D_{j_{k}}$ denotes the total derivative of order $k=\# J$ corresponding to the multi-index $J=\left(j_{1}, \ldots, j_{k}\right)$.

As a consequence of this result, the coefficients of $\varphi_{J}^{\alpha}$ of (5.3) can be computed via the standard prolongation formula

$$
\varphi_{J}^{\alpha}\left(x, u^{(k)}\right)=D_{J}\left[Q^{\alpha}\left(x, u^{(1)}\right)\right]+\sum_{i=1}^{p} \xi^{i}(x, u) u_{J, i}^{\alpha}
$$

which was first explicitly given in [29]. The next result appears in [30; Lemma 5.12].

Proposition 5.5. An evolutionary vector field $\mathbf{v}_{e v}$ commutes with the total derivative operators:

$$
\left[\mathbf{v}_{e v}, D_{i}\right]=0, \quad i=1, \ldots, p
$$

Remark: The only other vector fields on $\mathrm{J}^{\infty}$ which satisfy (5.9) are the constant coefficient independent variable vector fields $\sum c_{j} \partial_{x^{j}}$. Thus, the commutation property (5.9) almost completely characterizes evolutionary vector fields.

Let us now introduce a basis $\mathbf{v}_{1}, \ldots, \mathbf{v}_{r}$ for the infinitesimal generators $\mathfrak{g}$ of our transformation group. 
Definition 5.6. The Lie matrix of order $n$ is defined as the $r \times q^{(n)}$ matrix

$$
\mathbf{L}_{n}\left(x, u^{(n)}\right)=\left(\begin{array}{ccccccccc}
\xi_{1}^{1} & \ldots & \xi_{1}^{p} & \varphi_{1}^{1} & \ldots & \varphi_{1}^{q} & \ldots & \varphi_{J, 1}^{\alpha} & \ldots \\
\vdots & \ddots & \vdots & \vdots & \ddots & \vdots & \ddots & \vdots & \ddots \\
\xi_{r}^{1} & \ldots & \xi_{r}^{p} & \varphi_{r}^{1} & \ldots & \varphi_{r}^{q} & \ldots & \varphi_{J, r}^{\alpha} & \ldots
\end{array}\right)
$$

where $0 \leq \# J \leq n$. The entries $\xi_{\kappa}^{i}$, and $\varphi_{J, \kappa}^{\alpha}$ of (5.10) are the coefficients of the $n^{\text {th }}$ order prolongations $\operatorname{pr}^{(n)} \mathbf{v}_{\kappa}, \kappa=1, \ldots, r$, of the basis infinitesimal generators of $G$. Similarly, the Lie characteristic matrix of order $n$ is defined as the $r \times q^{(n)}$ matrix

$$
\mathbf{M}_{n}\left(x, u^{(n+1)}\right)=\left(\begin{array}{ccccccccc}
\xi_{1}^{1} & \ldots & \xi_{1}^{p} & Q_{1}^{1} & \ldots & Q_{1}^{q} & \ldots & D_{J} Q_{1}^{\alpha} & \ldots \\
\vdots & \ddots & \vdots & \vdots & \ddots & \vdots & \ddots & \vdots & \ddots \\
\xi_{r}^{1} & \ldots & \xi_{r}^{p} & Q_{r}^{1} & \ldots & Q_{r}^{q} & \ldots & D_{J} Q_{r}^{\alpha} & \ldots
\end{array}\right)
$$

where $0 \leq \# J \leq n$. The entries of (5.11) consist of the independent variable coefficients, the characteristics, and their total derivatives up to order $n$.

Proposition 5.7. The dimension of the orbit of $G^{(n)}$ through the point $z^{(n)} \in \mathrm{J}^{n}$ is the common Lie matrix rank

$$
\operatorname{rank} \mathbf{L}_{n}\left(z^{(n)}\right)=\operatorname{rank} \mathbf{M}_{n}\left(z^{(n+1)}\right)
$$

Here $z^{(n+1)}$ is any point with $\pi_{n}^{n+1}\left(z^{(n+1)}\right)=z^{(n)}$, since the rank of $\mathbf{M}_{n}$ is independent of the derivative coordinates $u_{K}^{\alpha}$ of order $\# K=n+1$.

Proof: The dimension of the orbit through $z^{(n)} \in \mathrm{J}^{n}$ is equal to the dimension of the subspace spanned by the prolonged infinitesimal generators, i.e., $\left.\left.\mathfrak{g}^{(n)}\right|_{z^{(n)}} \subset \mathrm{J}^{n}\right|_{z^{(n)}}$, and hence equals the rank of the Lie matrix $\mathbf{L}_{n}\left(z^{(n)}\right)$. Furthermore, the prolongation formula (5.8) implies that $\mathbf{M}_{n}\left(z^{(n+1)}\right)$ can be obtained from $\mathbf{L}_{n}\left(z^{(n)}\right)$ by adding suitable multiples of the first $p$ columns to the other columns. Since column operations do not alter the rank of a matrix, the proof is complete.

Q.E.D.

We can characterize the singular subset by the infinitesimal condition

$$
\mathcal{S}^{n}=\left\{z^{(n)} \in \mathrm{J}^{n}\left|\operatorname{dim} \mathfrak{g}^{(n)}\right|_{z^{(n)}}<s\right\} .
$$

Thus, the regular and singular subsets of $\mathrm{J}^{n}$ are determined by the common rank of Lie matrices (5.10), (5.11).

Corollary 5.8. The stable orbit dimension of $G$ is given by

$$
s=\max \left\{\operatorname{rank} \mathbf{L}_{n}\left(z^{(n)}\right) \mid z^{(n)} \in \mathrm{J}^{n}, n \geq 0\right\} .
$$

Consequently, a point $z^{(n)} \in \mathcal{S}^{n}$ is singular if and only if

$$
\operatorname{rank} \mathbf{L}_{n}\left(z^{(n)}\right)=\operatorname{rank} \mathbf{M}_{n}\left(z^{(n+1)}\right)<s, \quad \text { where } \quad \pi_{n}^{n+1}\left(z^{(n+1)}\right)=z^{(n)}
$$


In the particular case of planar curves, $p=q=1$, the Lie matrix $\mathbf{L}_{r-2}\left(x, u^{(r-2)}\right)$ of order $n=r-2=(\operatorname{dim} G)-2$ is square, and its determinant

$$
L\left(x, u^{(r-2)}\right)=\operatorname{det} \mathbf{L}_{r-2}\left(x, u^{(r-2)}\right)
$$

is known as the Lie determinant ${ }^{\dagger}$ of the transformation group $G$. See [31; Chapter 6] for a discussion, as well as a complete list of Lie determinants for the different finite-dimensional planar transformation groups appearing in Lie's classification. The Lie determinant equation is the differential equation of lowest order that admits the given transformation group as a symmetry group. In Sections 7 and 8 we will establish an algebraic method for constructing its general solution.

Example 5.9. The planar equi-affine group (3.9) has infinitesimal generators

$$
\mathbf{v}_{1}=\partial_{x}, \quad \mathbf{v}_{2}=\partial_{u}, \quad \mathbf{v}_{3}=x \partial_{u}, \quad \mathbf{v}_{4}=-x \partial_{x}+u \partial_{u}, \quad \mathbf{v}_{5}=-u \partial_{x}
$$

Applying the prolongation formula (5.3), (5.8), we compute the $n^{\text {th }}$ order Lie matrix to be

$$
\mathbf{L}_{n}\left(x, u^{(n)}\right)=\left(\begin{array}{ccccccc}
1 & 0 & 0 & 0 & 0 & \ldots & 0 \\
0 & 1 & 0 & 0 & 0 & \ldots & 0 \\
0 & x & 1 & 0 & 0 & \ldots & 0 \\
-x & u & 2 u_{x} & 3 u_{x x} & 4 u_{x x x} & \ldots & (n+1) u_{n} \\
-u & 0 & u_{x}^{2} & 3 u_{x} u_{x x} & 4 u_{x} u_{x x x}+3 u_{x x}^{2} & \ldots & P_{n}
\end{array}\right)
$$

where

$$
P_{n}\left(x, u^{(n)}\right)=\frac{1}{2} \sum_{i=1}^{n}\left(\begin{array}{c}
n \\
i
\end{array}\right) u_{i} u_{n-i+1}, \quad u_{n}=D_{x}^{n} u
$$

The Lie determinant is $L\left(x, u^{(3)}\right)=9 u_{x x}^{3}$, whose vanishing describes the singular subset $\mathcal{S}^{3}$. The verification that $\mathcal{S}^{n}$ for $n \geq 3$ is given by (3.15) is immediate from the form of the Lie matrix. The general connection between the Lie determinant and the higher order singular subvarieties will be discussed below.

\section{Singularities and Effectiveness.}

We are now ready to discuss the detailed geometry of the singular subsets of the jet spaces corresponding to a given transformation group $G$. The regular subset $\mathcal{V}^{n} \subset \mathrm{J}^{n}$ is nonempty and open provided $n$ is greater than or equal to the stabilization order of $G$. In the analytic category, $\mathcal{V}^{n}$ is dense in $\mathrm{J}^{n}$. However, at any given point $z \in M$, this does not necessarily imply that the jet fiber $\left.\mathrm{J}^{n}\right|_{z}$ contains any regular jets.

Definition 6.1. A point $z \in M$ is totally singular if $\left.\mathrm{J}^{n}\right|_{z} \subset \mathcal{S}^{n}$ for all $n$. A point which is not totally singular will be called totally regular.

$\dagger$ This is not quite correct if $G$ does not act transitively on an open subset of $\mathrm{J}^{r-2}$, which occurs when either $G$ acts intransitively on $\mathbb{R}^{2}$ or its prolonged orbit dimensions pseudostabilize. See $[\mathbf{3 1}]$ for the appropriate modifications in such cases. 
Thus, a point $z \in M$ is totally regular provided $\left.\mathrm{J}^{n}\right|_{z}$ contains a regular jet for $n$ sufficiently large. The set $M_{0} \subset M$ consisting of all totally regular points forms a nonempty open submanifold. Totally singular points are rather pathological. The following examples show that they can occur for non-analytic actions.

Example 6.2. Consider the one-parameter group (4.4) acting on $M=\mathbb{R}^{2}$. The stable orbit dimension is $s(M)=1$, and so every point in the right half plane $\{x \leq 0\}$ is totally singular. On the other hand, the restricted action on the open submanifold $W=\{x<0\}$ is completely trivial, so $s(W)=0$, and every point in $W$ now becomes totally regular. Thus, for smooth actions, the notion of a totally singular point can change upon restriction of the group action to an open submanifold.

Example 6.3. Let

$$
\mathbf{h}_{1}(x)= \begin{cases}e^{-1 /|x|}, & x \neq 0, \\ 0, & x=0 .\end{cases}
$$

Consider the two-parameter abelian transformation group

$$
(x, u) \longmapsto\left(x+a \mathbf{h}_{1}(u)+b, u\right), \quad \text { where } \quad(a, b) \in G=\mathbb{R}^{2} .
$$

The orbits are the horizontal lines, and so $G$ acts regularly. Since the infinitesimal generators are $\mathbf{v}_{1}=\mathbf{h}_{1}(u) \partial_{x}, \mathbf{v}_{2}=\partial_{x}$, the $n^{\text {th }}$ order Lie matrix is

$$
\mathbf{L}_{n}\left(x, u^{(n)}\right)=\left(\begin{array}{ccccc}
\mathbf{h}_{1}(u) & 0 & -\mathbf{h}_{1}^{\prime}(u) u_{x}^{2} & -\mathbf{h}_{1}^{\prime \prime}(u) u_{x}^{3}-3 \mathbf{h}_{1}^{\prime}(u) u_{x} u_{x x} & \cdots \\
1 & 0 & 0 & 0 & \cdots
\end{array}\right),
$$

with the $(1, k+2)$ entry being $\mathbf{h}_{1}(u) D_{x}^{k+1} u-D_{x}^{k}\left[\mathbf{h}_{1}(u) u_{x}\right]$ for $0 \leq k \leq n$. Consequently, the singular subset has two components,

$$
\mathcal{S}^{n}=\{u=0\} \cup\left\{u_{x}=u_{x x}=\cdots=u_{n}=0\right\} \subset \mathrm{J}^{n},
$$

and every point on the $x$ axis is totally singular.

Such pathologies cannot occur in the analytic category.

Theorem 6.4. If $G$ acts analytically, then there are no totally singular points.

Proof: The proof rests on a series of lemmas. Let $z_{0} \in M$. We introduce local coordinates $z=(x, u)$ near $z_{0}=(0,0)$, which we take as the origin for simplicity. A vector field

$$
\mathbf{v}=\sum_{i=1}^{m} \zeta^{i}(z) \frac{\partial}{\partial z^{i}}
$$

is said to be of degree $n$ at $z_{0}$ if all its coefficients and their partial derivatives up to order $n-1$ vanish at $z_{0}=0$, so $\partial_{J} \zeta^{i}(0)=0$ for $0 \leq \# J<n$. In particular, the isotropy subalgebra of $z_{0}$ consists of all infinitesimal generators that have degree 1 (or more) at $z_{0}$. The condition that a vector field have a certain degree at a point is, in fact, independent of the choice of local coordinates. 
Lemma 6.5. If a vector field $\mathbf{v}$ has degree $n+1$ at $z_{0}$, then its $n^{\text {th }}$ order prolongation $\operatorname{pr}^{(n)} \mathbf{v}$ vanishes on $\left.\mathrm{J}^{n}\right|_{z_{0}}$.

A vector field $\mathbf{v}$ is called a polynomial vector field of degree $n$ if all its coefficients are polynomials of degree $\leq n$ in the given local coordinates $z=(x, u)$. A polynomial vector field $\mathbf{v}$ is said to be homogeneous of degree $n$ if its coefficients $\zeta^{i}$ are homogeneous polynomials of degree $n$. For example, the elementary scaling vector field

$$
\mathbf{s}=\sum_{i=1}^{m} z^{i} \frac{\partial}{\partial z^{i}}=\sum_{j=1}^{p} x^{j} \frac{\partial}{\partial x^{j}}+\sum_{\alpha=1}^{q} u^{\alpha} \frac{\partial}{\partial u^{\alpha}}
$$

is homogeneous of degree 1. Any analytic vector field can thus be written as a convergent power series whose $n^{\text {th }}$ term is a homogeneous polynomial vector field of degree $n$ at the point $z_{0}=0$.

Lemma 6.6. Let $\mathbf{v}$ be a polynomial vector field of degree $n$. Then $\left.\operatorname{pr}^{(n)} \mathbf{v}\right|_{z^{(n)}}=0$ for all $\left.z^{(n)} \in \mathrm{J}^{n}\right|_{z_{0}}$ if and only if $\mathbf{v}=p_{n-1}(z) \mathbf{s}$ is a multiple of the scaling vector field (6.2) where the coefficient $p_{n-1}(z)$ is a scalar-valued homogeneous polynomial of degree $n-1$.

Proof: Unfortunately, at present the only proof I know is a straightforward, but tedious computation, based on the local coordinate formulae (5.3), (5.8) for the prolongations. For brevity, I shall omit the details.

Q.E.D.

Lemma 6.7. Let $\mathbf{v}$ be a smooth vector field on $M$. Suppose that $\left.\operatorname{pr}^{(n)} \mathbf{v}\right|_{z^{(n)}}=0$ for all $\left.z^{(n)} \in \mathrm{J}^{n}\right|_{z_{0}}$. Then $\mathbf{v}=p_{n-1}(z) \mathbf{s}+\mathbf{v}_{n+1}$, where $p_{n-1}(z)$ is a scalar-valued homogeneous polynomial of degree $n-1$ and $\mathbf{v}_{n+1}$ is a vector field of degree $n+1$.

Proof: We use Taylor's theorem to write $\mathbf{v}=\mathbf{w}_{n}+\mathbf{v}_{n+1}$, where $\mathbf{w}_{n}$ is a polynomial vector field of degree $n$, and the remainder $\mathbf{v}_{n+1}$ has degree $n+1$. The lemma then follows immediately from Lemmas 6.5 and 6.6.

Q.E.D.

A simple induction on the order $n$ now demonstrates that the only analytic vector field that vanishes on all jet spaces at the point $z_{0}$ is the trivial one.

Lemma 6.8. Let $\mathbf{v}$ be an analytic vector field on $M$. Suppose that $\left.\operatorname{pr}^{(n)} \mathbf{v}\right|_{z^{(n)}}=0$ for all $\left.z^{(n)} \in \mathrm{J}^{n}\right|_{z_{0}}$ and all $n>0$. Then $\mathbf{v} \equiv 0$.

Theorem 6.4 now follows immediately from this final lemma.

Q.E.D.

Ovsiannikov's stabilization theorem [36], [31; Theorem 5.11], completely characterizes the stable orbit dimension. The usual statement of this result contains the hypothesis that the group acts locally effectively, and the conclusion is that the stable orbit dimension equals the dimension of $G$. However, this is not quite justified, as the following example makes clear.

$\dagger$ With a little more work, this condition can be relaxed to just require $n>n_{0}$ for some $n_{0}$. 
Example 6.9. Consider the two-parameter abelian transformation group $G \simeq \mathbb{R}^{2}$ acting on $M=\mathbb{R}^{2}$ via $(x, u) \mapsto\left(x, u+a \mathbf{h}_{0}(x)+b \mathbf{h}_{0}(-x)\right)$, where $(a, b) \in G,(x, u) \in M$. The $C^{\infty}$ function $\mathbf{h}_{0}$ is defined in (4.3). The only group transformation which fixes every point $(x, u)$ is the identity $a=b=0$, and so $G$ acts effectively on $M$. However, it is not hard to see that the stable orbit dimension is $s=1$, which is strictly less than the dimension of $G$. Note also that the $u$-axis consists entirely of totally singular points.

The problem with this example is that $G$ acts effectively on $M$, but does not act effectively on either the right half plane $\{x>0\}$ or left half plane $\{x<0\}$. In other words, at least in the smooth category, a group may act effectively on a manifold, but not effectively on certain open submanifolds, and this would cause the traditional statement of the stabilization theorem to be invalid. In order to state a correct smooth version of the theorem, we need a slight refinement of the notion of effectiveness.

Definition 6.10. A transformation group $G$ acts effectively on subsets if $G_{W}^{*}=\{e\}$ for every open $W \subset M$. The group acts locally effectively on subsets if $G_{W}^{*}$ is a discrete subgroup of $G$ for every open $W \subset M$.

Clearly, if $G$ acts effectively on subsets, then $G$ acts effectively. While the converse does not hold for general smooth actions, it is true in the analytic category.

Lemma 6.11. If $G$ acts analytically on $M$ and acts (locally) effectively, then $G$ acts (locally) effectively on subsets.

The proof is a straightforward application of analytic continuation, based on the fact that the only analytic function $\psi: M \rightarrow M$ which agrees with the identity map on an open subset of $M$ is the identity. We can now state a correct version of the stabilization theorem.

Theorem 6.12. A Lie group $G$ acts locally effectively on subsets of $M$ if and only if for every open submanifold $W \subset M$, the stable orbit dimension of the restricted action of $G$ on $W$ equals the dimension of the group: $s(W)=s(M)=r=\operatorname{dim} G$.

Proof: The proof is adapted from that in [31], which works as long as we are not at a totally singular point. First, if $G$ does not act locally effectively on $M$, then $G^{(n)}$ does not act locally effectively on $\mathrm{J}^{n}(M, p)$, and hence the maximal orbit dimension $s_{n}$ is strictly less than the dimension of $G$. This implies that the stable orbit dimension is strictly less than the dimension of $G$, so $s(M)<r$ in this case. The same clearly holds for any open $W \subset M$.

To prove the converse, suppose that $G$ acts locally effectively on subsets of $M$, but there exists an open $W \subset M$ for which the stable orbit dimension of $G$ restricted to $W$ satisfies $s=s(W)<r$. Let $z_{0} \in W$ be a totally regular point, so that for $n$ sufficiently large, there exists a regular jet $\left.z_{0}^{(n)} \in \mathrm{J}^{n}\right|_{z_{0}}$, which requires that $\left.\operatorname{dim} \mathfrak{g}^{(n)}\right|_{z^{(n)}}=s$ for all $z^{(n)}$ in a neighborhood of $z_{0}^{(n)}$. We can therefore choose a basis $\mathbf{v}_{1}, \ldots, \mathbf{v}_{r}$ of the Lie algebra $\mathfrak{g}$ such that the corresponding first $s$ prolonged infinitesimal generators $\operatorname{pr}^{(n)} \mathbf{v}_{1}, \ldots, \operatorname{pr}^{(n)} \mathbf{v}_{s}$ form a basis for $\left.\mathfrak{g}^{(n)}\right|_{z^{(n)}}$ at each $z^{(n)} \in \mathcal{W}^{n}$ belonging to some connected, open neighborhood 
$\mathcal{W}^{n} \subset \mathrm{J}^{n}$ of $z_{0}^{(n)}$. This implies that, on $\mathcal{W}^{n}$, the remaining infinitesimal generators can be written as linear combinations

$$
\operatorname{pr}^{(n)} \mathbf{v}_{\mu}=\sum_{\nu=1}^{s} \lambda_{\mu}^{\nu} \operatorname{pr}^{(n)} \mathbf{v}_{\nu}, \quad \mu=s+1, \ldots, r
$$

of the basis generators. The coefficients $\lambda_{\mu}^{\nu}\left(z^{(n)}\right), \nu=1, \ldots, s, \mu=s+1, \ldots, r$, are uniquely determined $n^{\text {th }}$ order differential functions of $z^{(n)} \in \mathcal{W}^{n}$. If we can prove that the coefficients $\lambda_{\mu}^{\nu}$ are, in fact, constants, then Theorem 6.12 follows. Indeed, if we apply the projection $\pi_{0}^{n}: \mathrm{J}^{n} \rightarrow M$ to (6.3), we deduce that the $r-s$ infinitesimal generators $\mathbf{v}_{\mu}-\sum_{\nu=1}^{s} \lambda_{\mu}^{\nu} \mathbf{v}_{\nu}$ vanish identically in the open neighborhood $\mathcal{W}^{0}=\pi_{0}^{n}\left(\mathcal{W}^{n}\right)$ of $z_{0}$, which would contradict our original assumption that $G$ acts locally effectively on subsets of $M$.

Clearly, the prolonged vector fields $\mathrm{pr}^{(m)} \mathbf{v}_{1}, \ldots, \mathrm{pr}^{(m)} \mathbf{v}_{s}$ remain linearly independent on the open subset $\mathcal{W}^{m}=\left(\pi_{n}^{m}\right)^{-1} \mathcal{W}^{n} \subset \mathrm{J}^{m}$. Since $s$ is the stable orbit dimension, if $m \geq n$, the prolonged vector fields $\mathrm{pr}^{(m)} \mathbf{v}_{1}, \ldots, \mathrm{pr}^{(m)} \mathbf{v}_{s}$ must form a basis for $\left.\mathfrak{g}^{(m)}\right|_{z^{(m)}}$ for any $z^{(m)} \in \mathcal{W}^{m}$. Therefore, the linear identities (6.3) will continue to hold for all higher order prolongations of the generators, and hence, on $\mathcal{W}^{m}$,

$$
\operatorname{pr} \mathbf{v}_{\mu}=\sum_{\nu=1}^{s} \lambda_{\mu}^{\nu} \operatorname{pr} \mathbf{v}_{\nu}, \quad \mu=s+1, \ldots, r,
$$

for the same uniquely determined differential functions $\lambda_{\mu}^{\nu}\left(z^{(n)}\right)$. Indeed, if we apply $d \pi_{n}^{m}$ to (6.4), both sides will project to their counterparts in (6.3), and hence by uniqueness, the coefficients must be the same.

We now apply the evolutionary projection $\pi_{e v}$ to $(6.4)$, to obtain

$$
\mathbf{w}_{\mu}=\sum_{\nu=1}^{s} \lambda_{\mu}^{\nu} \mathbf{w}_{\nu}, \quad \mu=s+1, \ldots, r,
$$

where $\mathbf{w}_{\kappa}=\pi_{e v}\left(\mathrm{pr} \mathbf{v}_{\kappa}\right)$ denotes the evolutionary part of $\mathrm{pr} \mathbf{v}_{\kappa}$. Taking the commutator of (6.5) with the total derivative $D_{i}$ and using the property (5.9) of evolutionary vector fields yields

$$
0=\sum_{\nu=1}^{s} D_{i}\left(\lambda_{\mu}^{\nu}\right) \mathbf{w}_{\nu}, \quad \mu=s+1, \ldots, r .
$$

Linear independence of the vector fields $\mathrm{pr} \mathbf{v}_{1}, \ldots, \mathrm{pr} \mathbf{v}_{s}$ implies linear independence of their evolutionary representatives $\mathbf{w}_{1}, \ldots, \mathbf{w}_{s}$. Therefore

$$
D_{i}\left(\lambda_{\mu}^{\nu}\right)=0, \quad i=1, \ldots, p, \quad \nu=1, \ldots, s, \quad \mu=s+1, \ldots, r,
$$

which implies that all the coefficients $\lambda_{\mu}^{\nu}$ are constant.

Q.E.D.

An immediate consequence of this result is that, assuming local effectiveness, the set of totally regular points is dense and open in $M$. Of course, in the analytic category, Theorem 6.4 implies that every point is totally regular, and so this result is only of interest in more pathological smooth cases. 
Corollary 6.13. If $G$ acts locally effectively on subsets of $M$, then the set of totally singular points contains no open subset of $M$.

Another immediate consequence of the stabilization Theorem 6.12 is the local freeness of the prolonged action on the regular subset of jet space.

Theorem 6.14. If $G$ acts locally effectively on subsets, then $G$ acts locally freely on the regular subset $\mathcal{V}^{n} \subset \mathrm{J}^{n}$. Therefore, in such cases, the singular subset is given by

$$
\mathcal{S}^{n}=\left\{z^{(n)} \in \mathrm{J}^{n}\left|\operatorname{dim} \mathfrak{g}^{(n)}\right|_{z^{(n)}}<r\right\}=\left\{z^{(n)} \in \mathrm{J}^{n} \mid \operatorname{rank} \mathbf{L}_{n}\left(z^{(n)}\right)<r\right\} .
$$

Corollary 6.15. If $G$ acts analytically and locally effectively on subsets, then an $n^{\text {th }}$ order moving frame exists in a neighborhood of a point $z^{(n)} \in \mathrm{J}^{n}$ if and only if $z^{(n)} \in \mathcal{V}^{n}$ is a regular jet. In particular, the group admits locally equivariant moving frames of any order greater than or equal to the stabilization order.

It would be nice to know a nonlocal counterpart of Theorem 6.14: if $G$ acts effectively on subsets, then $G^{(n)}$ acts freely on (a dense open subset of) $\mathcal{V}^{n}$ provided $n$ is sufficiently large. This is particularly important for our moving frame constructions, since the theorem only guarantees a locally equivariant moving frame. However, it seems highly unlikely, particularly in the analytic category, that an effectively acting group acts only locally freely on a dense open subset of $\mathcal{V}^{n}$ for all $n$. One approach to proving such a result would be to find a direct, "non-infinitesimal" proof of the stabilization Theorem 6.12. The following interesting example, due to J. Pohjanpelto [37], shows that smooth actions can admit regular jets, of arbitrarily high order, where the prolonged group action is not free. It is not clear whether such pathology can occur in the analytic category.

Example 6.16. Let $M=\mathbb{R} \times S^{1}$ with coordinates $(x, u)$, where $0 \leq u<2 \pi$. The one-parameter group

$$
(x, u) \longmapsto\left(x, u+\varepsilon\left[1+\mathbf{h}_{1}(x)\right] \bmod 2 \pi\right), \quad \varepsilon \in \mathbb{R},
$$

where $\mathbf{h}_{1}$ is given in (6.1) acts effectively on subsets. The orbits are the one-dimensional vertical circles, and $G=\mathbb{R}$ acts locally freely on $M$, and freely on $M \backslash U_{0}$, where $U_{0}=$ $\{x=0\}$ denotes the circle through the origin. Therefore, every point in $\mathrm{J}^{n}=\mathcal{V}^{n}$ is regular. However, the action is only locally free on $\mathrm{J}^{n} \mid U_{0}$ for any $n \geq 0$. Indeed, since all the derivatives of $\mathbf{h}_{1}(x)$ vanish at the origin the prolonged action on $\mathrm{J}^{n} \mid U_{0}$ reduces to

$$
\left(0, u, u_{x}, \ldots, u_{n}\right) \longmapsto\left(0, u+\varepsilon \bmod 2 \pi, u_{x}, \ldots, u_{n}\right),
$$

and so the isotropy subgroup for any $z^{(n)}=\left(0, u, u_{x}, \ldots, u_{n}\right) \in \mathrm{J}^{n} \mid U_{0}$ is the discrete subgroup $2 \pi \mathbb{Z} \subset \mathbb{R}$ consisting of integral multiples of $2 \pi$. Note particularly that although $G^{(n)}$ acts freely on a dense open subset of $\mathrm{J}^{n}$, the set of points where the prolonged action is not free contains the entire jet fiber over the subvariety $U_{0}$.

An important application of Theorem 6.12 is the following "Wronskian lemma," generalizing the classical result on the linear dependence of functions with vanishing Wronskian determinant. Applications to the problem of characterizing differential operators, both 
linear and nonlinear, which leave a prescribed finite-dimensional subspace of functions invariant, and the consequent method of constructing explicit solutions to partial differential equations by reduction to dynamical systems are discussed in [21].

Definition 6.17. A set of vector-valued functions $h_{\kappa}: X \rightarrow \mathbb{R}^{q}, \kappa=1, \ldots, r$, is linearly dependent on a subset $W \subset X$ if there exist constants $c=\left(c_{1}, \ldots, c_{r}\right) \neq 0$, not all zero, such that

$$
\sum_{\kappa=1}^{r} c_{\kappa} h_{\kappa}(x) \equiv 0, \quad \text { for all } \quad x \in W .
$$

The functions are linearly independent on $W$ if the only constant coefficient linear combination that vanishes identically is the trivial one $c_{1}=\cdots=c_{r}=0$. The functions $h_{1}, \ldots, h_{r}$ are linearly independent on subsets of $X$ if they are linearly independent on every open subset $W \subset X$.

In the analytic category, linear independence implies linear independence on subsets. On the other hand, the smooth functions $\mathbf{h}_{0}(x)$ and $\mathbf{h}_{0}(-x)$, cf. (4.3), are linearly independent on $\mathbb{R}$ but not linearly independent on subsets. Standard existence and uniqueness theorems imply that linearly independent solutions of a linear system of ordinary differential equations are always linearly independent on subsets.

Classically, one checks the linear independence of scalar functions by analyzing their Wronskian determinant. In the multivariate version, one needs to analyze the ranks of general rectangular Wronskian matrices.

Definition 6.18. The $n^{\text {th }}$ order Wronskian matrix of smooth functions $h_{\kappa}: X \rightarrow \mathbb{R}^{q}$, $\kappa=1, \ldots, r$, where $X \subset \mathbb{R}^{p}$, is the $r \times q\left(\begin{array}{c}p+n \\ n\end{array}\right)$ matrix

$$
\mathbf{W}_{n}(x)=\left(\begin{array}{cccccc}
h_{1}^{1}(x) & \ldots & h_{1}^{q}(x) & \ldots & \partial_{J} h_{1}^{\alpha}(x) & \ldots \\
\vdots & \ddots & \vdots & \ddots & \vdots & \ddots \\
h_{r}^{1}(x) & \ldots & h_{r}^{q}(x) & \ldots & \partial_{J} h_{r}^{\alpha}(x) & \ldots
\end{array}\right)
$$

whose entries are the partial derivatives of the $h$ 's with respect to the $x$ 's of all orders $0 \leq \# J \leq n$.

Theorem 6.19. A set of functions $h_{\kappa}: X \rightarrow \mathbb{R}^{q}, \kappa=1, \ldots, r$, is linearly independent on subsets of $X$ if and only if their $(r-1)^{\text {st }}$ order Wronskian matrix $\mathbf{W}_{r-1}(x)=$ $\mathbf{W}_{r-1}\left[h_{1}, \ldots, h_{r}\right](x)$ has maximal rank $r$ for all $x$ in a dense open subset of $X$.

Proof: Consider the action

$$
(x, u) \longmapsto\left(x, u+\sum_{\kappa=1}^{r} t_{\kappa} h_{\kappa}(x)\right), \quad t=\left(t_{1}, \ldots, t_{r}\right) \in \mathbb{R}^{r},
$$

of the abelian $r$-parameter Lie group $G=\mathbb{R}^{r}$. The infinitesimal generators are the vector fields

$$
\mathbf{v}_{\kappa}=\sum_{\alpha=1}^{q} h_{\kappa}^{\alpha}(x) \frac{\partial}{\partial u^{\alpha}}
$$


The Lie matrix $\mathbf{L}_{n}\left(z^{(n)}\right)$ of this group consists of $p$ initial columns of zeros, since the group acts trivially on the independent variables, followed by the Wronskian matrix $\mathbf{W}_{n}(x)$ of the functions $h_{\kappa}$. Therefore, the prolonged orbit dimension $s_{n}$ equals the maximal rank of $\mathbf{W}_{n}(x)$. It is not hard to see that $G$ acts locally effectively on subsets if and only if the functions $h_{\kappa}$ are linearly independent on subsets. Therefore, Theorem 6.12 implies that, on any open subset $Y \subset X$, we have $r=\max \operatorname{rank} \mathbf{W}_{n}(x)$ for $x \in Y$ and any $n$ greater than or equal to the stabilization order of the transformation group (6.9). Moreover, it is not hard to prove that the group (6.9) does not pseudostabilize, and hence Theorem 4.2 implies that the stabilization order is at most $r-1$.

Q.E.D.

For example, in the case $p=q=1$, so that $h_{1}(x), \ldots, h_{r}(x)$ are scalar-valued functions of a single variable $x$, the matrix $\mathbf{W}_{r-1}\left[h_{1}, \ldots, h_{r}\right]$ becomes the usual $r \times r$ Wronskian of the functions $h_{\kappa}$. Therefore, Theorem 6.19 reduces to the statement that a set of scalar functions is linearly independent on subsets of $\mathbb{R}$ if and only if its Wronskian determinant does not vanish identically, thus providing a converse to the standard Wronskian lemma in the theory of linear ordinary differential equations.

Remark: Kolchin [23; p. 86] proves a version of Theorem 6.19 assuming that the functions belong to a differential field, e.g., the field of meromorphic functions. Further extensions appear in $[\mathbf{2 1}]$.

Since the group (6.9) does not pseudostabilize, its stabilization order is the minimal $n$ for which maxrank $\mathbf{W}_{n}(x)=\max \operatorname{rank} \mathbf{W}_{n+1}(x)=s$. Thus $s=r$ if and only if the functions are linearly independent on subsets. In the smooth category, they may be linearly independent even when $s<r$. In the analytic category, $s$ equals the dimension of the vector space spanned by $h_{1}, \ldots, h_{r}$.

Suppose that $h_{1}(x), \ldots h_{r}(x)$ are linearly independent on subsets. By definition, a totally singular point is a point $x \in X$ such that $\operatorname{rank} \mathbf{W}_{n}(x)<r$ for all $n \geq 0$; the remaining points are called totally regular. Theorem 6.19 states that the set of totally regular points is open, dense in $X$. Moreover, if the functions are analytic, then linear independence implies linear independence on subsets, and every point is totally regular. At a totally regular point $x \in X$, we have, by definition, rank $\mathbf{W}_{n}(x)=r$ for $n$ sufficiently large (and not necessarily $\leq r$ ), which, by continuity, also holds in a neighborhood of $x$. However, even in the analytic category, it may be impossible to find a finite value of $n$ for which rank $\mathbf{W}_{n}(x)=r$ for all $x \in X$. For example, let $h_{1}(x)$ be an analytic function which has a zero of order $k$ at $x=a_{k}$, for $k=1,2,3, \ldots$, where $a_{k} \rightarrow \infty$ as $k \rightarrow \infty$; such a function can be constructed using a Weierstrass product expansion, cf. [3; p. 194]. Then rank $\mathbf{W}_{n}\left[h_{1}\right]\left(a_{k}\right)=0$ for $n<k$, while $\operatorname{rank} \mathbf{W}_{n}\left[h_{1}\right](x)=1$ for $n \geq k$ and $x$ in any neighborhood of $a_{k}$ that does not contain $a_{k+1}, a_{k+2}, \ldots$

\section{Singular Submanifolds.}

We now turn to the study of singular submanifolds. From now on we make the blanket assumption that $G$ is an $r$-dimensional Lie group that acts locally effectively on subsets of $M$. Theorem 6.12 implies that its stable orbit dimension equals its dimension, $s=r=\operatorname{dim} G$. The regular subset $\mathcal{V}^{n} \subset \mathrm{J}^{n}$ consists of all orbits whose dimension is the same as the dimension of $G$. 
Definition 7.1. A submanifold $S \subset M$ is order $n$ regular if $\mathrm{j}_{n} S \subset \mathcal{V}^{n}$. A submanifold $S$ is called regular if it is regular at some finite order $n$. A submanifold $S \subset M$ is totally singular at a point $z \in S$ if $\left.\mathrm{j}_{n} S\right|_{z} \subset \mathcal{S}^{n}$ for all $n=0,1, \ldots$ A totally singular submanifold consists entirely of totally singular points.

In other words, if $S$ is an order $n$ regular submanifold, then $G^{(n)}$ acts locally freely in a neighborhood of $\mathrm{j}_{n} S \subset \mathcal{V}^{n}$. Note that if $S$ is order $n$ regular, then it is also order $m$ regular for any $m \geq n$. The set of regular points on a submanifold forms an open submanifold $S_{0} \subset S$, which is a dense subset if $G$ acts analytically and $S$ is an analytic submanifold. The regular submanifolds of order $n$ are precisely those that admit (locally equivariant) moving frames of that order. Vice versa, a totally singular submanifold admits no moving frame of any order.

The goal of this section is to provide a direct, geometrical characterization of totally singular submanifolds. The first easy result demonstrates that non-freeness of the isotropy subgroup of a submanifold at a point implies singularity.

Proposition 7.2. Let $S$ be a submanifold, and $G_{S} \subset G$ its isotropy subgroup. If $S$ is regular at $z \in S$, then $G_{S}$ acts locally freely on $S$ at $z$.

Proof: Assume that $G_{S}$ does not act locally freely at a point $z \in S$. This implies that there exists a nontrivial infinitesimal generator $0 \neq \mathbf{v} \in \mathfrak{g}_{S}$ that vanishes at $z$, so $\left.\mathbf{v}\right|_{z}=0$. Therefore, $\exp (t \mathbf{v}) z=z$ and $\exp (t \mathbf{v}) S \subset S$. Together, these imply that, for every $n$, the $n$-jet $z^{(n)}=\left.\mathrm{j}_{n} S\right|_{z}$ is a fixed point for the prolonged one-parameter group, so $\exp (t \mathbf{v})^{(n)} \cdot z^{(n)}=z^{(n)}$. But this implies $\left.\operatorname{pr}^{(n)} \mathbf{v}\right|_{z^{(n)}}=0$, and so $z^{(n)} \in \mathcal{S}^{n}$ for all $n$, proving that $S$ is totally singular at $z$.

Q.E.D.

Example 7.3. Consider the analytic two-parameter group $(x, u) \mapsto(\lambda x, u+b)$. The infinitesimal generators are $\mathbf{v}_{1}=\partial_{u}, \mathbf{v}_{2}=x \partial_{x}$, and hence the Lie matrix is

$$
\left(\begin{array}{cccccc}
0 & 1 & 0 & 0 & 0 & \cdots \\
x & 0 & -u_{x} & -2 u_{x x} & -3 u_{x x x} & \cdots
\end{array}\right) .
$$

This shows that the singular subset is

$$
\mathcal{S}^{n}=\left\{x=u_{x}=u_{x x}=\cdots=u_{n}=0\right\} .
$$

Therefore, any horizontal line $L=\{u=c\}$ is totally singular when $x=0$, but is regular at nonzero $x$. The isotropy subgroup $G_{L}$ consists of the scaling $x \mapsto \lambda x$, which does not act locally freely at $x=0$. On the other hand, the curve $C$ defined by the function

$$
u=\mathbf{h}_{2}(x)= \begin{cases}e^{-1 / x}, & x>0 \\ 0, & x=0 \\ x e^{1 / x}, & x<0\end{cases}
$$

has infinite order contact with the $x$ axis at the origin, and is totally singular at $z_{0}=(0,0)$. However, $C$ has trivial isotropy subgroup $G_{C}=\{e\}$. This shows that the converse to Proposition 7.2 is not necessarily true, at least in the smooth category. 
Example 7.4. Slightly generalizing the preceding example, we consider the analytic four-parameter group $(x, u) \mapsto(\lambda x+a, \mu u+b)$. The infinitesimal generators are

$$
\mathbf{v}_{1}=\partial_{x}, \quad \mathbf{v}_{2}=x \partial_{x}, \quad \mathbf{v}_{3}=\partial_{u}, \quad \mathbf{v}_{4}=u \partial_{u},
$$

and so the Lie matrix is

$$
\left(\begin{array}{ccccccc}
1 & 0 & 0 & 0 & 0 & 0 & \cdots \\
x & 0 & -u_{x} & -2 u_{x x} & -3 u_{x x x} & -4 u_{x x x x} & \cdots \\
0 & 1 & 0 & 0 & 0 & 0 & \cdots \\
0 & u & u_{x} & u_{x x} & u_{x x x} & u_{x x x x} & \cdots
\end{array}\right) .
$$

Therefore, the singular subset occurs where all but one of the derivatives $u_{x}, u_{x x}, u_{x x x}, \ldots$ vanish. Therefore, any straight line $u=a x+b$ is totally singular at every point thereon. Furthermore, the polynomial curves $u=a\left(x-x_{0}\right)^{n}+u_{0}$ for $n \geq 2$, are totally singular at the point $\left(x_{0}, u_{0}\right)$. Also, by reversing the roles of $x$ and $u$, the curves $x=\left(u-u_{0}\right)^{n}+x_{0}$ are also totally singular at $\left(x_{0}, u_{0}\right)$. Thus, each point $z_{0} \in \mathbb{R}^{2}$ has an interesting collection of totally singular analytic curves passing through it.

Theorem 7.5. Suppose $G$ acts analytically. An analytic submanifold $S$ is regular at a point $z_{0} \in S$ if and only if its isotropy subgroup $G_{S}$ acts locally freely on $S$ at $z_{0}$.

Proof: Assume that $S$ is totally singular at a point $z_{0} \in S$. This assumption means that we can find a nontrivial infinitesimal generator $0 \neq \mathbf{v} \in \mathfrak{g}$ such that $\left.\operatorname{pr}^{(n)} \mathbf{v}\right|_{z_{0}}=0$ for all $n \geq 0$. Choose local coordinates $(x, u)$ such that $S=\{u=f(x)\}$ is transverse at $z_{0}=\left(x_{0}, f\left(x_{0}\right)\right)$. The prolongation formula (5.8) implies that the total derivatives of the characteristic $Q\left(x, u^{(1)}\right)$ of $\mathbf{v}$ all vanish at $z_{0}$, so that $D_{J} Q\left(x_{0}, \mathrm{j}_{k+1} f\left(x_{0}\right)\right)=0$ for all $0 \leq k=\# J$, where $\left(x_{0}, \mathrm{j}_{k+1} f\left(x_{0}\right)\right)=\left.\mathrm{j}_{k+1} S\right|_{z_{0}}$. This means that the analytic function $F(x)=Q\left(x, \mathrm{j}_{1} f(x)\right)$ satisfies $\partial_{J} F(x)=0$ for all $J$, and hence, by analyticity, $F(x) \equiv 0$ for all $x$. Consequently, $u=f(x)$ is a solution to the invariant surface conditions $Q\left(x, u^{(1)}\right)=0$ for the vector field $\mathbf{v}$. Proposition 5.2 implies that $\mathbf{v}$ is an infinitesimal generator of the isotropy subgroup $G_{S}$. Moreover, since we have $\left.\mathbf{v}\right|_{z_{0}}=0$, the isotropy subgroup $G_{S}$ cannot act locally freely on $S$ at $z_{0}$.

Q.E.D.

As we saw, in the smooth category, freeness of the isotropy subgroup action on $S$ is not enough to prevent isolated totally singular points from occurring. The next result shows that if all the points on $S$ are totally singular, then the isotropy subgroup must act non-freely, even in the smooth category. The method of proof differs from that of the analytic Theorem 7.5, and is based on the proof of the stabilization Theorem 6.12 .

Theorem 7.6. Let $G$ act smoothly and locally effectively on subsets of $M$. A submanifold $S \subset M$ is totally singular if and only if its isotropy subgroup $G_{S}$ does not act locally freely on $S$.

Proof: Assume that $G_{S}$ does act locally freely. Let

$$
t_{n}=\max \left\{\left.\operatorname{dim} \mathfrak{g}^{(n)}\right|_{z^{(n)}} \mid z^{(n)} \in \mathrm{j}_{n} S\right\} .
$$

Clearly, $t_{0} \leq t_{1} \leq \cdots$. Thus $t_{m}=t=\max t_{n}$ for all $m$ sufficiently large. Since $\mathrm{j}_{n} S \subset \mathcal{S}^{n}$ for all $n$, we have $t<r$. Let $n$ be the minimal order at which $t_{n}=t$. Let $R \subset S$ denote the 
open submanifold where $\left.\operatorname{dim} \mathfrak{g}^{(n)}\right|_{z^{(n)}}=t$ for all $z^{(n)} \in \mathrm{j}_{n} R$. Local freeness of the action of $G_{S}$ implies that we can choose a basis $\mathbf{v}_{1}, \ldots, \mathbf{v}_{r}$ of $\mathfrak{g}$ such that, in a neighborhood of $R$, (a) $\mathbf{v}_{1}, \ldots, \mathbf{v}_{s}$ are pointwise linearly independent, and span the Lie algebra $\mathfrak{g}_{S}$ of the isotropy group of $S$ (restricted to $R$ ), and

(b) the $n^{\text {th }}$ order prolongations $\mathrm{pr}^{(n)} \mathbf{v}_{1}, \ldots, \mathrm{pr}^{(n)} \mathbf{v}_{t}$ of the first $t$ generators are pointwise linearly independent on $\mathrm{j}_{n} R$.

Therefore,

$$
\left.\mathfrak{g}^{(m)}\right|_{z^{(m)}}=\operatorname{Span}\left\{\left.\operatorname{pr}^{(m)} \mathbf{v}_{1}\right|_{z^{(m)}}, \ldots,\left.\operatorname{pr}^{(m)} \mathbf{v}_{t}\right|_{z^{(m)}}\right\},
$$

for all $z^{(m)} \in \mathrm{j}_{m} R$ and all $m \geq n$, and hence the remaining generators can be written as linear combinations of the independent generators. Therefore, letting $m \rightarrow \infty$, we obtain the linear relations

$$
\operatorname{pr} \mathbf{v}_{t+\nu}=\sum_{\kappa=1}^{t} \lambda_{\nu}^{\kappa} \operatorname{pr} \mathbf{v}_{\kappa}, \quad \nu=1, \ldots, r-t .
$$

Projecting the higher order linear dependencies (7.2) on $\mathrm{J}^{n}$ implies that the coefficients $\lambda_{\nu}^{\kappa}=\lambda_{\nu}^{\kappa}\left(z^{(n)}\right)$ are functions of only the $n^{\text {th }}$ order jet $z^{(n)} \in \mathrm{j}_{n} R$.

The remainder of the proof is almost identical to that of Theorem 6.12. We can introduce local coordinates $(x, u)$ on $M$ such that $R$ is (locally) the the graph of a section $u=f(x)$. Applying the evolutionary projection $\pi_{e v}$ to (7.2) gives

$$
\mathbf{w}_{t+\nu}=\sum_{\kappa=s+1}^{t} \lambda_{\nu}^{\kappa} \mathbf{w}_{\kappa}, \quad \nu=1, \ldots, r-t,
$$

on $R$, where $\mathbf{w}_{\kappa}=\pi_{e v}\left(\operatorname{pr} \mathbf{v}_{\kappa}\right)$ denotes the evolutionary part of $\operatorname{pr} \mathbf{v}_{\kappa}$. Proposition 5.2 implies that the first $s$ evolutionary vector fields $\mathbf{w}_{1}, \ldots, \mathbf{w}_{s}$ vanish on $R$ because they lie in its isotropy subalgebra. Taking the commutator of (7.3) with the total derivative $D_{i}$ and using the property (5.9) of evolutionary vector fields yields

$$
0=\sum_{\kappa=s+1}^{t} D_{i}\left(\lambda_{\nu}^{\kappa}\right) \mathbf{w}_{\kappa}, \quad \nu=1, \ldots, r-t,
$$

which holds on $R$. Linear independence of the vector fields $\operatorname{pr} \mathbf{v}_{\kappa}$ on the submanifold $R$ implies linear independence of their evolutionary representatives $\mathbf{w}_{s+1}, \ldots, \mathbf{w}_{t}$ on $R$. Therefore

$$
D_{i}\left(\lambda_{\nu}^{\kappa}\right)=0, \quad i=1, \ldots, p,
$$

on $R$, which implies that all the coefficients $\lambda_{\nu}^{\kappa}$ are constant on $R$. Consequently, the nonzero vector field

$$
\widetilde{\mathbf{v}}_{\nu}=\mathbf{v}_{\nu}-\sum_{\kappa=s+1}^{t} \lambda_{\nu}^{\kappa} \mathbf{v}_{\kappa}
$$

vanishes on $R$. This contradicts our original assumption that $G_{S}$ acts locally freely on $S$, since $\widetilde{\mathbf{v}}_{\nu}$ belongs to its isotropy subalgebra.

Q.E.D. 
Example 7.7. Consider the action $z \mapsto A z+b$ of the full affine group $\mathrm{A}(2)$ on $M=\mathbb{R}^{2}$. Here the totally singular curves are the parabolas and the straight lines. The isotropy group of a parabola, say $u=x^{2}$, is the two-dimensional non-abelian subgroup

$$
(x, u) \mapsto\left(\lambda x+\mu, \lambda^{2} u+2 \lambda \mu x+\mu^{2}\right) .
$$

In this case the singular subset of $\mathrm{J}^{n}$ is governed by the total derivatives of the affine Lie determinant

$$
\mathcal{V}^{n}=\left\{\left(x, u^{(n)}\right) \mid D_{x}^{n-4}\left[u_{x x} u_{x x x x}-\frac{5}{3} u_{x x x}^{2}\right]=0\right\} .
$$

Note that parabolas and straight lines comprise the general solution to the affine Lie determinant equation

$$
u_{x x} u_{x x x x}=\frac{5}{3} u_{x x x}^{2} .
$$

Remark: In both of the special and full affine groups, if an analytic curve contains one totally singular point, then it is totally singular at all points. However, the scaling group in Example 7.4 does not enjoy this property. This is related to the factorizability of the Lie determinant associated with the group, although I do not know a general geometrical characterization of this phenomenon.

In the planar case, the Lie determinant can be used to completely characterize the totally singular curves. This theorem also serves to explain why higher order singular subsets are usually obtained by total differentiation (or prolongation) of the Lie determinant equation.

Theorem 7.8. Suppose $G$ is an $r$-dimensional Lie group that acts transitively and locally effectively on subsets on a two-dimensional manifold. Assume that the prolonged group actions do not pseudostabilize. Then, locally, the set of totally singular curves form the general solution to the Lie determinant equation $L\left(x, u^{(r-2)}\right)=0$.

Remark: The result remains valid in the intransitive and/or pseudostabilization cases if one suitably modifies the definition of the Lie determinant, as in [31].

Proof: Since the singular subset $\mathcal{S}^{r-2}=\left\{L\left(x, u^{(r-2)}\right)=0\right\}$ coincides with the Lie determinant ordinary differential equation, the latter admits $G$ as a symmetry group. Let $u=f(x)$ be a solution, and suppose the jet $z_{0}^{(r-2)}=\left(x_{0}, f^{(r-2)}\left(x_{0}\right)\right)$ is a regular (nonsingular) point for the Lie determinant differential equation, meaning that we can uniquely and smoothly solve the equation $L\left(x, u^{(r-2)}\right)=0$ for the highest order derivative $u_{r-2}$ in a neighborhood of $z_{0}^{(r-2)}$. (Under the hypotheses, the regular points form a dense open subset of $\mathcal{S}^{r-2}$ - this can be seen from the Lie classification of Lie determinants given in [31].) Let $0 \neq \mathbf{v} \in \mathfrak{g}$ be an infinitesimal generator whose prolongation $\operatorname{pr}^{(r-2)} \mathbf{v}$ vanishes at $z_{0}^{(r-2)}$; such a generator exists because $z_{0}^{(r-2)}$ lies in $\mathcal{S}^{r-2}$. The corresponding one-parameter subgroup $\exp t \mathbf{v}$ then fixes $z_{0}^{(r-2)}$. On the other hand, since $G$ is a symmetry group of the Lie determinant equation, it maps solutions to solutions. By the uniqueness of solutions to ordinary differential equations, $\exp t \mathbf{v}$ also fixes the entire solution $u=f(x)$, and hence $G$ does not act locally freely on the curve. Proposition 7.2 completes the proof.

Q.E.D. 
The same proof will extend to curves in higher dimensional spaces, or more general submanifolds, provided we have a uniqueness theorem for the solutions to the differential equation defined by the singular subset $\mathcal{S}^{n}$ for some $n$. This would follow if we knew the differential system $\mathcal{S}^{n} \subset \mathrm{J}^{n}$ was of finite type for $n$ sufficiently large; see [18]. This seems a reasonable conjecture, although I do not know any results along these lines. The following example shows that there are some subtleties involved in trying to formulate a general theorem.

Example 7.9. Consider the four parameter group

$$
(x, u, v) \longmapsto\left(\lambda x+a, \lambda^{3} u+\mu x+b, \lambda^{2} v\right),
$$

acting on $M=\mathbb{R}^{3}$ with infinitesimal generators

$$
\mathbf{v}_{1}=\partial_{x}, \quad \mathbf{v}_{2}=\partial_{u}, \quad \mathbf{v}_{3}=x \partial_{u}, \quad \mathbf{v}_{4}=x \partial_{x}+3 u \partial_{u}+2 v \partial_{v} .
$$

We consider curves in $M$, viewing $x$ as the independent variable and $u, v$ as dependent variables. The second order Lie matrix is

$$
\left(\begin{array}{ccccccc}
1 & 0 & 0 & 0 & 0 & 0 & 0 \\
0 & 1 & 0 & 0 & 0 & 0 & 0 \\
0 & x & 0 & 1 & 0 & 0 & 0 \\
x & 3 u & 2 v & 2 u_{x} & v_{x} & u_{x x} & 0
\end{array}\right) .
$$

Therefore, the first two singular subsets are

$$
\mathcal{S}^{1}=\left\{v=v_{x}=0\right\} \subset \mathrm{J}^{1}, \quad \mathcal{S}^{2}=\left\{v=v_{x}=u_{x x}=0\right\} \subset \mathrm{J}^{2} .
$$

The differential system $\mathcal{S}^{1}$ is underdetermined, and so solutions to its initial value problem are not unique. However, $\mathcal{S}^{2}$ is a normal system of ordinary differential equations, and hence, applying the proof of Theorem 7.8, we conclude that the totally singular curves are the solutions to the system $u_{x x}=0, v=0$, which implies that $u=a x+b, v=0$. Interestingly, since $\operatorname{dim} \mathrm{J}^{1}>\operatorname{dim} G$, the first order Lie matrix already has more columns than rows, and one might have guessed, in analogy with the scalar case, that it would govern the totally singular curves. This example indicates that the finite type conjecture is a bit more subtle than might be initially expected.

\section{Homogeneous Spaces.}

In the case $G$ acts transitively on $M$, then we can locally ${ }^{\dagger}$ identify $M$ with the homogeneous space $M \simeq G / H$. Here $H=G_{z_{0}}$ is the isotropy subgroup of the base point $z_{0}=\pi(e)$, which is the image of the identity group element under the natural projection $\pi: G \rightarrow G / H$. In this final section, we conduct a more detailed investigation into the totally singular submanifolds $S \subset G / H$. We shall relate them to the existence of certain Lie subgroups of the transformation group $G$.

$\dagger$ Recall that $G$ might only be a local transformation group. 
Note that we can always move any submanifold $S \subset M$ by a group transformation without affecting its intrinsic geometric properties. By transitivity, then, we can assume that, without loss of generality, the submanifold passes through the base point $z_{0}=\pi(e)$. We first analyze the easier case when the totally singular submanifold has an isotropy subgroup that acts transitively on it.

Definition 8.1. A submanifold $S \subset M$ is transitively totally singular if its isotropy subgroup $G_{S}$ acts transitively and non-freely on $S$.

Remark: In the formulation of this definition, we are excluding those smooth submanifolds which contain a totally singular point, but nevertheless have freely acting isotropy subgroups. Theorem 7.5 shows that such pathology does not occur in the analytic category.

If $K=G_{S}$ is the isotropy subgroup of a submanifold $S \subset G / H$ passing through $z_{0}=\pi(e)$, and $K$ acts transitively on $S$, then we can identify $S=\pi(K)$. Conversely, if $S=\pi(K)$ for some subgroup $K$, then $K$ acts transitively on $S$, and hence is a subgroup of the full isotropy subgroup of $S$. We require a general characterization of the full isotropy subgroup, which relies on the following purely group-theoretic constructions.

Recall that a subgroup $K \subset G$ is called self-normalizing provided $g^{-1} \cdot K \cdot g=K$ if and only if $g \in K$. The Cartan subgroups of a semisimple Lie group are particularly important examples [38]. The characterization of transitively totally singular submanifolds relies on a generalization of the notion of a self-normalizing subgroup.

Definition 8.2. Let $H \subset G$ be a subgroup of a group $G$. The $H$-normalizer of a subgroup $K \subset G$, denoted $\mathcal{N}_{H}(K)$, consists of all group elements $g \in(K \cdot H) \cap(H \cdot K)$ such that

$$
g^{-1} \cdot K \cdot g \subset K \cdot H, \quad \text { and } \quad g \cdot K \cdot g^{-1} \subset K \cdot H .
$$

The subgroup $K$ is called self $H$-normalizing if it equals its $H$-normalizer: $K=\mathcal{N}_{H}(K)$.

Note that we are not assuming that $K \cdot H$ is a subgroup here, which would require $K$. $H=H \cdot K$. Unfortunately, I have been unable to find a purely Lie algebraic characterization of the $H$-normalizer of a Lie subgroup.

Lemma 8.3. Consider the homogeneous space $\pi: G \rightarrow G / H$. Let $K \subset G$ be a subgroup, and let $S=\pi(K) \subset G / H$. Then the isotropy subgroup of $S$ equals the $H$ normalizer of $K$.

Proof: A group element $g \in G$ is a symmetry of $S=\pi(K)$ if and only if for every $k \in K$, we have

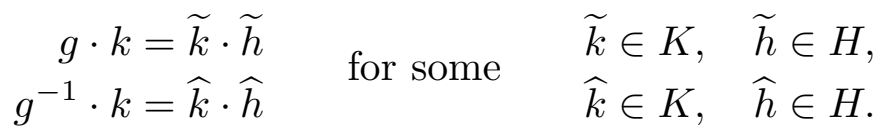

(The proof does rely on both conditions.) If we set $k=e$ in (8.2), we find

$$
g=k_{0} \cdot h_{0}=h_{1} \cdot k_{1} \quad \text { for some } \quad k_{0}, k_{1} \in K, \quad h_{0}, h_{1} \in H,
$$


so that $g \in(K \cdot H) \cap(H \cdot K)$. Therefore, given $k \in K$,

$$
\begin{aligned}
& g \cdot k \cdot g^{-1}=g \cdot\left(k \cdot k_{1}^{-1}\right) \cdot h_{1}^{-1}=\bar{k} \cdot \bar{h} \cdot h_{1}^{-1} \in K \cdot H, \\
& g^{-1} \cdot k \cdot g=g^{-1} \cdot\left(k \cdot k_{0}\right) \cdot h_{0}=k^{*} \cdot h^{*} \cdot h_{0} \in K \cdot H,
\end{aligned}
$$

for certain $\bar{k}, k^{*} \in K$ and $\bar{h}, h^{*} \in H$. In the second equalities, we use (8.2) with $k$ replaced by $k \cdot k_{1}^{-1}, k \cdot k_{0}$, respectively. This proves that the isotropy subgroup $G_{S} \subset \mathcal{N}_{H}(K)$ is contained in the $H$-normalizer of $K$.

Conversely, if $g=k_{0} \cdot h_{0}=k_{1} \cdot h_{1} \in(K \cdot H) \cap(H \cdot K)$ satisfies the $H$-normalizer equations (8.1), then for any $k \in K$ we have

$$
\begin{aligned}
& g \cdot k=g \cdot\left(k \cdot k_{1}\right) \cdot k_{1}^{-1}=\left[g \cdot\left(k \cdot k_{1}\right) \cdot g^{-1}\right] \cdot g \cdot k_{1}^{-1}=\bar{k} \cdot \bar{h} \cdot h_{0}^{-1}, \\
& g^{-1} \cdot k=g^{-1} \cdot\left(k \cdot k_{0}^{-1}\right) \cdot k_{0}=\left[g^{-1} \cdot\left(k \cdot k_{0}^{-1}\right) \cdot g\right] \cdot g^{-1} \cdot k_{0}=k^{*} \cdot h^{*} \cdot h_{0}^{-1},
\end{aligned}
$$

and hence $g$ satisfies (8.2), which implies that $g \in G_{S}$.

Q.E.D.

Example 8.4. Consider the action of the planar affine group $A(2)$ discussed in Example 7.7, which we identify with the homogeneous space $\pi: \mathrm{A}(2) \rightarrow \mathrm{A}(2) / \mathrm{GL}(2) \simeq$ $\mathbb{R}^{2}$ corresponding to the linear subgroup $\mathrm{GL}(2) \subset \mathrm{A}(2)$. Consider the two-dimensional subgroup $K$ given by the horizontal transformations $(x, u) \mapsto(\lambda x+\mu, u)$. Let us compute its GL(2)-normalizer using the symmetry criterion (8.2). We identify affine group elements $(A, b) \in \mathrm{A}(2)$ with $3 \times 3$ matrices $\left(\begin{array}{cc}A & b \\ 0 & 1\end{array}\right) \in \mathrm{GL}(3)$ in the usual manner. The subgroup $K$ consists of all matrices of the form $\left(\begin{array}{lll}\lambda & 0 & \mu \\ 0 & 1 & 0 \\ 0 & 0 & 1\end{array}\right)$. Then $g=\left(\begin{array}{lll}\alpha & \beta & a \\ \gamma & \delta & 0 \\ 0 & 0 & 1\end{array}\right) \in K \cdot \operatorname{GL}(2)$ belongs to $\mathcal{N}_{\mathrm{GL}(2)}(K)$ provided

$$
\left(\begin{array}{ccc}
\alpha & \beta & a \\
\gamma & \delta & 0 \\
0 & 0 & 1
\end{array}\right) \cdot\left(\begin{array}{ccc}
\lambda & 0 & \mu \\
0 & 1 & 0 \\
0 & 0 & 1
\end{array}\right)=\left(\begin{array}{ccc}
\alpha \lambda & \beta & \alpha \mu+a \\
\gamma \lambda & \delta & \gamma \mu \\
0 & 0 & 1
\end{array}\right) \in K \cdot \operatorname{GL}(2)
$$

which requires that its $(2,3)$ entry vanishes, and hence $\gamma=0$. Therefore, the GL(2)normalizer of $K$ is the subgroup $(x, u) \mapsto(\alpha x+\beta u+a, \delta u)$, which forms the full fourdimensional isotropy subgroup of the $x$ axis which is the image $S=\pi(K)$.

On the other hand, the subgroup $\widetilde{K}$ given in (7.4) is self $H$-normalizing. Using the matrix version $\widetilde{k}=\left(\begin{array}{ccc}\lambda & 0 & \mu \\ 2 \lambda \mu & \lambda^{2} & \mu^{2} \\ 0 & 0 & 1\end{array}\right) \in \widetilde{K}$ for its elements, we see that $g=\left(\begin{array}{ccc}\alpha & \beta & a \\ \gamma & \delta & a^{2} \\ 0 & 0 & 1\end{array}\right) \in$ $\widetilde{K} \cdot \mathrm{GL}(2)$ belongs to $\mathcal{N}_{\mathrm{GL}(2)}(\widetilde{K})$ provided the product $g \cdot \widetilde{k}$ belongs to $\widetilde{K} \cdot \mathrm{GL}(2)$, which requires that its $(2,3)$ entry equals the square of its $(1,3)$ entry:

$$
\left(\alpha \mu+\beta \mu^{2}+a\right)^{2}=\gamma \mu+\delta \mu^{2}+a^{2} .
$$

Clearly this holds if and only if $\beta=0, \delta=\alpha^{2}, \gamma=2 \alpha a$, and hence $g \in \widetilde{K}$ already. This reconfirms that the isotropy subgroup of the parabola $\pi(\widetilde{K})=\left\{u=x^{2}\right\}$ equals $\widetilde{K}=G_{P}$. 
Theorem 8.5. There is a one-to-one correspondence ${ }^{\dagger}$ between transitively totally singular $p$-dimensional submanifolds $S \subset G / H$ passing through $z_{0}=\pi(e)$ and connected $k$-dimensional self $H$-normalizing Lie subgroups $K \subset G$ such that the intersection $K \cap H$ has dimension $t=k-p \geq 1$.

Remark: In view of Theorem 7.8, these constructions provide a purely algebraic approach to the solution of the Lie determinant differential equation. Vice-versa, the Lie determinant equation provides a differential equation approach to the algebraic problem of determining the $H$-normalizers of subgroups $K \subset G$.

Proof: Given such a subgroup $K$, let $S=\pi(K)$. It is not hard to prove that $S$ is, in a neighborhood of $z_{0}$, a smooth submanifold of the correct dimension; see also Lemma 8.6 below. Moreover, $K$ is clearly contained in the isotropy subgroup of $S$, and acts non-freely since it has a nontrivial intersection with $H$. According to Lemma $8.3, K$ is the entire isotropy subgroup of $S$. Conversely, given a transitively totally singular submanifold $S$, passing through $z_{0}$, we let $K=G_{S}$ denote its isotropy subgroup. Transitivity implies that $S=\pi(K)$. Moreover, any $k \in K \cap H$ satisfies $k \cdot z_{0}=z_{0}$, and hence $K$ will act freely on $S$ at $z_{0}$ if and only if $K \cap H=\{e\}$.

Q.E.D.

The intransitive case is a little harder. We begin with a lemma that characterizes those submanifolds of $G$ which project to smooth submanifolds of the homogeneous space $G / H$. Let $L_{g}(h)=g \cdot h$ and $R_{g}(h)=h \cdot g$ denote the right and left multiplication maps on $G$. We let $\mathfrak{h} \subset \mathfrak{g}$ denote the Lie subalgebra for the subgroup $H$.

Lemma 8.6. If $N \subset G$ is a smooth $n$-dimensional submanifold, then $S=\pi(N)$ is, locally, a smooth $p$-dimensional submanifold of $M=G / H$ if and only if

$$
\operatorname{dim}\left[\left.\mathfrak{h}\right|_{e} \cap\left(d L_{g}\right)^{-1}\left(\left.T N\right|_{g}\right)\right]=n-p, \quad \text { for all } \quad g \in N .
$$

In other words, near $e$, a submanifold $N \subset G$ will project to a submanifold $S \subset G / H$ provided the subspace $\left.\mathfrak{h}\right|_{e} \cap\left(d L_{g}\right)^{-1}\left(\left.T N\right|_{g}\right)$ has constant dimension. Note that, globally, the projected submanifold $S=\pi(N)$ may have self-intersections.

Proof: By the Implicit Function Theorem, we need only to prove that $($ ker $d \pi) \cap T N$ has constant dimension $n-p$ over $N$. Recall that the isotropy subgroup of a point $z=\pi(g) \in M$ is $G_{z}=g \cdot H \cdot g^{-1}$, with Lie algebra $\mathfrak{g}_{z}=\operatorname{Ad} g(\mathfrak{h})$, where Ad denote the adjoint representation of $G$ on its Lie algebra [38]. Since we are identifying Lie algebra elements with right-invariant vector fields on $G$, we have

$$
\left.\operatorname{ker} d \pi\right|_{g}=\left.\operatorname{Ad} g(\mathfrak{h})\right|_{g}=\left.d L_{g} \circ\left(d R_{g}\right)^{-1} \mathfrak{h}\right|_{g}=d L_{g}\left(\left.\mathfrak{h}\right|_{e}\right) \text {. }
$$

In other words, the kernel of $d \pi$ equals the subspace of $\left.T G\right|_{g}$ spanned by the left-invariant vector fields corresponding to elements of the subalgebra $\mathfrak{h}$. Therefore,

$$
\left.\left(\left.\operatorname{ker} d \pi\right|_{g}\right) \cap T N\right|_{g}=\left.d L_{g}\left(\left.\mathfrak{h}\right|_{e}\right) \cap T N\right|_{g}=d L_{g}\left[\left.\mathfrak{h}\right|_{e} \cap\left(d L_{g}\right)^{-1}\left(\left.T N\right|_{g}\right)\right]
$$

$\dagger$ We identify two submanifolds passing through a point if they are the same in a neighborhood thereof, i.e., they have the same germ. 
will have the correct dimension if and only if (8.3) holds.

Q.E.D.

Remark: If $N=K$ is a Lie subgroup with Lie algebra $\mathfrak{k}$, then

$$
\left(d L_{g}\right)^{-1}\left(\left.T K\right|_{g}\right)=\left.\mathfrak{k}\right|_{e},
$$

and hence condition (8.3) is automatic. This justifies our earlier claim during the proof of Theorem 8.5.

Now, suppose $S=\pi(N)$ is a totally singular submanifold passing through $z_{0}=\pi(e)$. Let $K=G_{S}$ be the intransitively acting isotropy subgroup of $S$. In a neighborhood of $e \in G$, we can identify $N=K \cdot A \subset G$, where $e \in A \subset G$ is a submanifold that is transverse to $K \cdot H$, meaning that

$$
\left.T A\right|_{e} \cap\left(\left.\mathfrak{k}\right|_{e}+\left.\mathfrak{h}\right|_{e}\right)=\{0\} .
$$

Now, for $g=k \cdot a \in N$ near $e$,

$$
\left.T N\right|_{g}=\left.\mathfrak{k}\right|_{g}+d L_{k}\left(\left.T A\right|_{a}\right)
$$

Since

$$
\left(d L_{g}\right)^{-1}\left(\left.\mathfrak{k}\right|_{g}\right)=\operatorname{Ad} g^{-1}\left(\left.\mathfrak{k}\right|_{e}\right)=\operatorname{Ad} a^{-1}\left(\left.\mathfrak{k}\right|_{e}\right)
$$

we have

$$
\begin{aligned}
\left(d L_{g}\right)^{-1}\left(\left.T N\right|_{g}\right) & =\left(d L_{g}\right)^{-1}\left(\left.\mathfrak{k}\right|_{g}\right)+\left(d L_{a}\right)^{-1}\left(\left.T A\right|_{a}\right) \\
& =\operatorname{Ad} a^{-1}\left(\left.\mathfrak{k}\right|_{e}\right)+\left(d L_{a}\right)^{-1}\left(\left.T A\right|_{a}\right) .
\end{aligned}
$$

Now, by continuity, (8.4) implies that $\left(d L_{a}\right)^{-1}\left(\left.T A\right|_{a}\right)$ remains transverse to the subspace $\left.\mathfrak{k}\right|_{e}+\left.\left.\mathfrak{h}\right|_{e} \subset T G\right|_{e}$ for $a$ sufficiently close to the identity. Therefore

$$
\operatorname{dim}\left[\left.\left(d L_{g}\right)^{-1}\left(\left.T N\right|_{g}\right) \cap \mathfrak{h}\right|_{e}\right]=\operatorname{dim}\left[\operatorname{Ad} a^{-1}(\mathfrak{k}) \cap \mathfrak{h}\right], \quad \text { where } \quad g=k \cdot a .
$$

Consequently, Lemma 8.6 implies that $S=\pi(N)$ will be a smooth submanifold near $z_{0}$ if and only if

$$
\operatorname{dim}\left[\operatorname{Ad} a^{-1}(\mathfrak{k}) \cap \mathfrak{h}\right]=\operatorname{dim} \mathfrak{k} \cap \mathfrak{h},
$$

for $a$ near $e$. Continuous dependence of $\operatorname{Ad} a(\mathbf{v})$ on $a$ for fixed $\mathbf{v} \in \mathfrak{k} \cap \mathfrak{h}$ implies that (8.5) will hold if and only if $A \subset \mathcal{N}_{H}(K \cap H)$, where

$$
\mathcal{N}_{H}(K \cap H)=\left\{g \in G \mid g^{-1} k g \in H \text { for all } k \in K \cap H\right\}
$$

is the $H$-normalizer of the subgroup $K \cap H$. Finally, the group elements in $K=G_{S}$ that fix $z_{0}$ are those belonging to $K \cap H$, and hence the requirement that the isotropy subgroup does not act locally freely necessitates that the dimension (8.5) be at least 1 . We have therefore proved the following characterization of general totally singular submanifolds.

Theorem 8.7. Every submanifold $S \subset G / H$ which is totally singular at a point $z=\pi(g) \in S$ has the form $S=g \cdot S_{0}$, where $z=g \cdot z_{0}$, and we can locally identify $S_{0}=\pi(K \cdot A)$, where $K \subset G$ is a $k$-dimensional subgroup of $G$, with $t=\operatorname{dim} K \cap H \geq 1$, and $A \subset \mathcal{N}_{H}(K \cap H)$ is an l-dimensional submanifold. The dimension of $S$ is $p=k+l-t$, and $K \subset G_{S}$ is a non-freely acting subgroup of its isotropy group. 
Example 8.8. Consider the group $G=\mathbb{R}^{+} \ltimes \mathbb{R}^{2}$ with multiplication rule

$$
(\lambda, a, b) \cdot(\mu, c, d)=\left(\lambda \mu, a+\lambda c, b+\lambda^{2} c\right) .
$$

The group acts on $M=\mathbb{R}^{2}=G / H$ according to $(x, u) \mapsto\left(\lambda x+a, \lambda^{2} u+b\right)$; the isotropy subgroup is $H=G_{0}=\{(\lambda, 0,0)\}$. The straight line $L=\{u=0\}$ is totally singular. We can realize $L=\pi(K)$, where the isotropy subgroup $G_{L}=K=\{(\lambda, a, 0)\}$ acts transitively on $L$ and has one-dimensional intersection with $H$, confirming Theorem 8.5 in this case. Similarly, the parabola $P=\left\{u=x^{2}\right\}$ has the scaling subgroup $H$ as its isotropy subgroup, $G_{P}=H$, which is not transitive, and only the origin is a totally singular point. Indeed, $P=\pi(H \cdot A)$, where the curve $A=\left\{\left(1, a, a^{2}\right)\right\} \subset G$ is transverse to $H$, reconfirming Theorem 8.7.

Acknowledgments: It is a pleasure to thank Mark Fels, Niky Kamran, Juha Pohjanpelto, and Michael Singer for encouragement, critical comments, references, and suggestions. I would also like to acknowledge the support of the Mathematical Sciences Research Institute, in Berkeley, where the final parts of this research were completed.

\section{References}

[1] Ackerman, M., and Hermann, R., Sophus Lie's 1880 Transformation Group Paper, Math Sci Press, Brookline, Mass., 1975.

[2] Ackerman, M., and Hermann, R., Sophus Lie's 1884 Differential Invariant Paper, Math Sci Press, Brookline, Mass., 1976.

[3] Ahlfors, L.V., Complex Analysis, Second Edition, McGraw-Hill Book Co., New York, 1966.

[4] Berchenko, I.A., and Olver, P.J., Symmetries of polynomials, J. Symb. Comp., to appear.

[5] Budd, C.J., and Iserles, A., Geometric integration: numerical solution of differential equations on manifolds, Phil. Trans. Roy. Soc. London A 357 (1999), 945-956.

[6] Calabi, E., Olver, P.J., Shakiban, C., Tannenbaum, A., and Haker, S., Differential and numerically invariant signature curves applied to object recognition, Int. J. Computer Vision 26 (1998), 107-135.

[7] Calabi, E., Olver, P.J., and Tannenbaum, A., Affine geometry, curve flows, and invariant numerical approximations, Adv. in Math. 124 (1996), 154-196.

[8] Cartan, É., La Méthode du Repère Mobile, la Théorie des Groupes Continus, et les Espaces Généralisés, Exposés de Géométrie No. 5, Hermann, Paris, 1935.

[9] Cartan, É., La Théorie des Groupes Finis et Continus et la Géométrie Différentielle Traitées par la Méthode du Repère Mobile, Cahiers Scientifiques, Vol. 18, Gauthier-Villars, Paris, 1937.

[10] Cartan, É., Les problèmes d'équivalence, in: Oeuvres Complètes, Part. II, Vol. 2, Gauthier-Villars, Paris, 1953, pp. 1311-1334. 
[11] Dorodnitsyn, V.A., Finite difference models entirely inheriting continuous symmetry of original differential equations, Int. J. Mod. Phys. C 5 (1994), 723-734.

[12] Fels, M., and Olver, P.J., Moving coframes. I. A practical algorithm, Acta Appl. Math. 51 (1998), 161-213.

[13] Fels, M., and Olver, P.J., Moving coframes. II. Regularization and theoretical foundations, Acta Appl. Math. 55 (1999), 127-208.

[14] Fels, M., and Olver, P.J., Moving frames and moving coframes, preprint, University of Minnesota, 1997.

[15] Golubitsky, M., Stewart, I., and Schaeffer, D.G., Singularities and Groups in Bifurcation Theory, Vol. 2, Springer-Verlag, New York, 1988.

[16] Griffiths, P.A., On Cartan's method of Lie groups and moving frames as applied to uniqueness and existence questions in differential geometry, Duke Math. J. 41 (1974), 775-814.

[17] Griffiths, P.A., and Harris, J., Principles of Algebraic Geometry, John Wiley \& Sons, New York, 1978.

[18] Griffiths, P.A., and Jensen, G.R., Differential Systems and Isometric Embeddings, Annals of Math. Studies, No. 114, Princeton Univ. Press, Princeton, N.J., 1987.

[19] Guggenheimer, H.W., Differential Geometry, McGraw-Hill, New York, 1963.

[20] Jensen, G.R., Higher Order Contact of Submanifolds of Homogeneous Spaces, Lecture Notes in Math., 610, Springer-Verlag, New York, 1977.

[21] Kamran, N., Milson, R., and Olver, P.J. , Invariant modules and the reduction of nonlinear partial differential equations to dynamical systems, Adv. in Math., to appear.

[22] Killing, W., Erweiterung der Begriffes der Invarianten von Transformationgruppen, Math. Ann. 35 (1890), 423-432.

[23] Kolchin, E.R., Differential Algebra and Algebraic Groups, Academic Press, New York, 1973.

[24] Lie, S., Theorie der Transformationsgruppen I, Math. Ann. 16 (1880), 441-528; also Gesammelte Abhandlungen, Vol. 6, B.G. Teubner, Leipzig, 1927, pp. 1-94; see [1] for an English translation.

[25] Lie, S., Über Differentialinvarianten, Math. Ann. 24 (1884), 537-578; also Gesammelte Abhandlungen, Vol. 6, B.G. Teubner, Leipzig, 1927, pp. 95-138; see [2] for an English translation.

[26] Lie, S., Klassifikation und Integration von gewöhnlichen Differentialgleichungen zwischen $x, y$, die eine Gruppe von Transformationen gestatten I, II, Math. Ann. 32 (1888), 213-281; also Gesammelte Abhandlungen, Vol. 5, B.G. Teubner, Leipzig, 1924, pp. 240-310.

[27] Milne-Thompson, L.M., The Calculus of Finite Differences, Macmilland and Co., Ltd., London, 1951.

[28] Moons, T., Pauwels, E., Van Gool, L., and Oosterlinck, A., Foundations of semi-differential invariants, Int. J. Comput. Vision 14 (1995), 25-48.

[29] Olver, P.J., Symmetry groups and group invariant solutions of partial differential equations, J. Diff. Geom. 14 (1979), 497-542. 
[30] Olver, P.J., Applications of Lie Groups to Differential Equations, Second Edition, Graduate Texts in Mathematics, vol. 107, Springer-Verlag, New York, 1993.

[31] Olver, P.J., Equivalence, Invariants, and Symmetry, Cambridge University Press, Cambridge, 1995.

[32] Olver, P.J., Pseudo-stabilization of prolonged group actions. I. The order zero case, J. Nonlinear Math. Phys. 4 (1997), 271-277.

[33] Olver, P.J., Classical Invariant Theory, Cambridge University Press, Cambridge, 1999.

[34] Olver, P.J., Joint invariant signatures, preprint, University of Minnesota, 1999.

[35] Olver, P.J., Geometric foundations of numerical algorithms and symmetry, preprint, University of Minnesota, 1999.

[36] Ovsiannikov, L.V., Group Analysis of Differential Equations, Academic Press, New York, 1982.

[37] Pohjanpelto, J., personal communication, 1998.

[38] Varadarajan, V.S., Lie Groups, Lie Algebras, and their Representations, Springer-Verlag, New York, 1984. 